\title{
Spectropolarimetry of the 2012 outburst of SN 2009ip: a bi-polar explosion in a dense, disc-like CSM
}

\author{
Emma Reilly, ${ }^{\star}{ }^{\star}$ Justyn R. Maund,${ }^{2} \dagger$ Dietrich Baade,${ }^{3}$ J. Craig Wheeler, ${ }^{4}$ \\ Peter Höflich, ${ }^{5}$ Jason Spyromilio, ${ }^{3}$ Ferdinando Patat ${ }^{3}$ and Lifan Wang ${ }^{6}$ \\ ${ }^{1}$ Astrophysics Research Centre, School of Mathematics and Physics, Queen's University Belfast, Belfast BT7 1NN, UK \\ ${ }^{2}$ Department of Physics and Astronomy, The University of Sheffield, Hicks Building, Hounsfield Road, Sheffield S3 7RH, UK \\ ${ }^{3}$ ESO - European Organisation for Astronomical Research in the Southern Hemisphere, Karl-Schwarzschild-Str. 2, D-85748 Garching b. München, Germany \\ ${ }^{4}$ Department of Astronomy and McDonald Observatory, The University of Texas, 1 University Station C1402, Austin, TX 78712-0259, USA \\ ${ }^{5}$ Department of Physics, Florida State University, Tallahassee, FL 32306-4350, USA \\ ${ }^{6}$ Department of Physics, Texas A\&M University, College Station, TX 77843-4242, USA
}

Accepted 2017 May 17. Received 2017 May 15; in original form 2017 January 30

\begin{abstract}
We present a sequence of eight spectropolarimetric observations monitoring the geometric evolution of the late phase of the major 2012 outburst of SN 2009ip. These were acquired with the Focal Reducer and Low Dispersion Spectrograph polarimeter mounted on European Southern Observatory VLT. The continuum was polarized at $0.3-0.8$ per cent throughout the observations, showing that the photosphere deviated substantially from spherical symmetry by $10-15$ per cent. Significant line polarization is detected for both hydrogen and helium at high velocities. The similarity in the polarized signal between these elements indicates that they form in the same location in the ejecta. The line polarization ( $p \sim 1-1.5$ per cent) at low velocities revealed the presence of a highly aspherical hydrogen- and helium-rich circumstellar medium (CSM). Monte Carlo simulations of the observed polarimetry were performed in an effort to constrain the shape of the CSM. The simulations imply that the polarimetry can be understood within the framework of a disc-like CSM inclined by $14^{\circ} \pm 2^{\circ}$ out of the line of sight, obscuring the photosphere only at certain epochs. The varying temporal evolution of polarization at high and low velocities indicated that the fast-moving ejecta expanded with a preferred direction orthogonal to that of the CSM.
\end{abstract}

Key words: techniques: polarimetric-circumstellar matter-stars: mass-loss-supernovae: general - supernovae: individual: SN 2009ip.

\section{INTRODUCTION}

There is a growing body of evidence that suggests that the supernova (SN) explosions at the end of the life of massive stars are not spherically symmetric events. The most notable evidence was the resolved asymmetric structure of SN 1987A, as observed in late-time Hubble Space Telescope (HST) imaging (Jansen \& Jakobsen 2001; Larsson et al. 2013; Sinnott et al. 2013). Complex aspherical morphologies have also been observed in Galactic supernova remnants (SNRs), including that of Cassiopeia A (Fesen et al. 2001; Wheeler, Maund \& Couch 2008; Milisavljevic \& Fesen 2013; Rest et al. 2011b). Light echoes of SNe (Crotts \& Yourdon 2008; Rest et al. 2011a; Sinnott et al. 2013) and nebular phase spectroscopy (Maeda et al.

^E-mail: ereilly528@qub.ac.uk

$\dagger$ Royal Society Research Fellow.
2008) have also been utilized to reveal departures from spherical symmetry. One of the most substantive indicators for asymmetry in the last decade has come from spectropolarimetry of extragalactic SNe. Polarimetry has shown that significant departures from spherical symmetry are present in all types of core-collapse SNe (CCSNe) (Wang \& Wheeler 2008). The observations showed that the polarization often increases with time as the core of the explosion becomes increasingly exposed (Wang et al. 1996; Leonard et al. 2006; Maund et al. 2007a, 2009; Chornock et al. 2010). This suggests that the explosion itself is asymmetric in nature.

In addition to being a powerful probe of the 3D structure of $\mathrm{SNe}$ ejecta, spectropolarimetry can also divulge information on the medium surrounding the progenitor stars. This is particularly relevant for the interaction-dominated Type IIn SNe. The spectra of Type IIn SNe are hydrogen-rich and characterized by narrow $\left(\sim 1000 \mathrm{~km} \mathrm{~s}^{-1}\right)$ emission lines, and are an indicator that the ejecta are strongly interacting with dense interstellar or circumstellar matter (Schlegel 1990; Filippenko 1997). They are thought to originate 
from the deaths of very massive stars that have lost a considerable amount of their envelope through episodic mass loss (Gal-Yam et al. 2007; Taddia et al. 2013, see also Smith 2014). Alternatively, they may also arise through thermonuclear explosions in a dense circumstellar medium (CSM, e.g. Taddia et al. 2012; Silverman et al. 2013; Leloudas et al. 2015; Fox et al. 2015; Inserra et al. 2016), while Mackey et al. (2014) proposed that the interaction of the ejecta of a red supergiant explosion with a photoionization-confined shell could also produce a Type IIn SN.

One of the most controversial and best studied objects of this class is SN 2009ip. It was discovered by Maza et al. (2009) in the spiral galaxy NGC 7259 in 2009 August and designated an SN before it was re-classified as the extreme brightening of a Luminous Blue Variable (LBV, Berger, Foley \& Ivans 2009; Miller et al. 2009). Serendipitous pre-explosion HST images dating from 1999 allowed Smith et al. (2010) and Foley et al. (2011) to estimate an initial mass of 50-60 M $\odot$. SN 2009ip then endured three more years of outbursts culminating in two spectacular eruptions in 2012 (Pastorello et al. 2013).

In 2012 July and August, SN 2009ip began to re-brighten once again. This occurred in two phases, the first outburst beginning on 2012 July 24 (2012a) reached a peak absolute magnitude of $M_{\mathrm{V}} \approx-14$ mag. A second re-brightening event began on 2012 September 23 (2012b) and took the peak magnitude to $M_{\mathrm{R}}=-18.5$ mag, similar to some CCSNe. This outburst was accompanied by evidence of material travelling at speeds of up to $15000 \mathrm{~km} \mathrm{~s}^{-1}$ and a spectrum that was reminiscent of the early phases of Type IIn $\mathrm{SNe}$ (Mauerhan et al. 2013; Pastorello et al. 2013). This led many to believe that SN 2009ip had finally undergone a terminal explosion. The high SN-like velocities, however, had previously been observed in 2011 (Pastorello et al. 2013) and the luminosity of the 2012a event was somewhat low for an SN explosion. These observations cast doubt on the SN explosion scenario. The curious case of SN 2009ip has now been joined by a handful of similar objects, including SN 2015bh (Ofek et al. 2016; Soker \& Kashi 2016; Elias-Rosa et al. 2016; Thöne et al. 2016), SN Hunt 248 (Kankare et al. 2015) and LSQ13zm (Tartaglia et al. 2016).

The final fate of SN 2009ip has been much debated in the literature. The proposed explanations for the 2012 outbursts fall into three scenarios: a terminal CCSN in either the 2012a or 2012b outbursts (Mauerhan et al. 2013; Smith, Mauerhan \& Prieto 2014; Mauerhan et al. 2014) or another 'impostor' event from the eruptions of an LBV star (Pastorello et al. 2013; Fraser et al. 2013; Margutti et al. 2014). An alternative possibility is that the 2012 events were the result of close encounters and the possible merger of an LBV star and a more compact companion (Kashi, Soker \& Moskovitz 2013; Soker \& Kashi 2013).

Despite the detailed studies of this object, there is still no clear consensus on whether the progenitor star survived the events of 2012. As of yet there has been no evidence of nucleosynthesis products in late-time spectra which would confirm core collapse (Fraser et al. 2013; Margutti et al. 2014; Fraser et al. 2015). Most recently, Thöne et al. (2015) have reported that the luminosity of SN 2009ip has now declined below that of the possible progenitor observed in 1999. This possibly favours the terminal SN explosion scenario, or suggests that the star was in outburst during those preexplosion observations.

Mauerhan et al. (2014) have previously published polarimetric observations of this outburst. The large continuum polarization they observe at the peak of the $2012 \mathrm{~b}$ event suggests a highly aspherical interaction zone, from which they infer a disc-like or toroidal CSM. Additionally, the ejecta are orthogonal to the plane of the CSM.
They propose that the small angular coverage of the CSM requires a large SN-like impact velocity to produce the observed luminosity.

In this paper, we present spectropolarimetric observations of the decline of the $2012 \mathrm{~b}$ event. Section 2 contains information on the observations and data reduction, and in Section 3, we briefly discuss the spectral evolution. The polarimetric results are then analysed in Section 4. In Section 5, we present a Monte Carlo simulation of the geometry of the CSM and present our conclusions in Section 6.

\section{OBSERVATIONS AND DATA REDUCTION}

Spectropolarimetric observations of SN 2009ip were commenced on 2012 November 7, $35 \mathrm{~d}$ after the UV maximum of the $2012 \mathrm{~b}$ event on 2012 October 3 (Margutti et al. 2014). An outline of the observations is given in Table 1 . In total, eight observations were conducted over six different epochs. A portion of these data, four spectra over three epochs, have previously been published in Mauerhan et al. (2014). These are marked in Table 1. Further observations are presented here, for those data previously published a different approach to the interstellar polarization (ISP) is taken. The spectropolarimetric data were acquired using the European Southern Observatory (ESO) Very Large Telescope (VLT) Antu telescope with the Focal Reducer and Low Dispersion Spectrograph (FORS2) instrument in the PMOS mode (Appenzeller et al. 1998). In PMOS mode, FORS 2 operates as a dual-beam optical spectropolarimeter. FORS2 measures linear polarization with a 'super achromatic' halfwave retarder plate that rotates the plane of polarization before a Wollaston prism separates the light into two orthogonally polarized beams. Observations were conducted with the retarder plate at four angles: $0.0,45.0,22.5$ and 67.5 . The 'striped' PMOS slit mask was used with slit width of $1^{\prime \prime}$. The $300 \mathrm{~V}$ and $1200 \mathrm{R}$ grisms were used in order to obtain a dispersion of $\sim 3.23 \AA$ pixel $^{-1}$ and a spectral resolution of $\sim 12 \AA$ across the spectrum with the former, whilst the latter provided a dispersion of $\sim 0.73 \AA \mathrm{pixel}^{-1}$ and a spectral resolution of $\sim 2.7 \AA$. To prevent second-order contamination at longer wavelengths an order separation filter (GG435) was used, yielding wavelength ranges of $4450-8650 \AA$ for the $300 \mathrm{~V}$ and $5750-7310 \AA$ for the 1200R grism.

The data were reduced in $\operatorname{IRAF}^{1}$ using the method outlined in Maund et al. (2007a) and the normalized Stokes parameters ( $q$ and $u$ ) were calculated following Patat \& Romaniello (2006) . In order to increase the signal-to-noise ratio $(\mathrm{S} / \mathrm{N})$, the spectra were binned to 15 and $5 \AA$ for the $300 \mathrm{~V}$ and $1200 \mathrm{R}$ spectra, respectively. This was performed prior to the calculation of the Stokes parameters.

The data were corrected for the wavelength-dependent chromatic zero-angle offset of the retarder plate, and the polarized spectra were debiased following the prescription of Quinn (2012). At each epoch, calibrated flux spectra were produced using the spectra observed at all four retarder plate angles. The combined spectrum was flux calibrated using observations of a flux standard star, observed with the polarimetry optics in place and with the retarder plate at $0^{\circ}$.

\section{SPECTRAL EVOLUTION}

The flux spectra for the observations taken with the FORS2 instrument between 2012 November $7(+35 \mathrm{~d})$ and December $25(+83 \mathrm{~d})$

\footnotetext{
${ }^{1}$ IRAF is distributed by the National Optical Astronomy Observatories, which are operated by the Association of Universities for Research in Astronomy, Inc., under cooperative agreement with the National Science Foundation http://iraf.noao.edu/.
} 
Table 1. Table of VLT FORS2 observations for SN 2009ip.

\begin{tabular}{|c|c|c|c|c|c|c|}
\hline Object & $\begin{array}{l}\text { Date } \\
\text { (UT) }\end{array}$ & Grism & $\begin{array}{c}\text { Exposure } \\
\text { (s) }\end{array}$ & Mean airmass & $\begin{array}{l}\text { Epoch }^{a} \\
\text { (d) }\end{array}$ & $\mathrm{S} / \mathrm{N}$ \\
\hline $\mathrm{SN} 2009 \mathrm{IP}^{b}$ & 2012117.02 & $300 \mathrm{~V}$ & $4 \times 330$ & 1.01 & +35 & 550 \\
\hline $\mathrm{SN}^{2009 \mathrm{IP}^{b}}$ & 2012117.03 & $1200 \mathrm{R}$ & $4 \times 470$ & 1.04 & +35 & 400 \\
\hline LTT1020 ${ }^{c}$ & 2012117.06 & $300 \mathrm{~V}$ & 10 & 1.12 & & \\
\hline LTT1020 ${ }^{c}$ & 2012117.06 & $1200 \mathrm{R}$ & 30 & 1.12 & & \\
\hline SN2009IP & 20121114.07 & $300 \mathrm{~V}$ & $4 \times 310$ & 1.17 & +42 & 375 \\
\hline SN2009IP & 20121114.08 & $1200 \mathrm{R}$ & $4 \times 500$ & 1.26 & +42 & 300 \\
\hline LTT $1020^{c}$ & 20121114.18 & $300 \mathrm{~V}$ & 10 & 1.06 & & \\
\hline LTT1020 & 20121114.19 & $1200 \mathrm{R}$ & 25 & 1.06 & & \\
\hline $\mathrm{SN}^{2009 \mathrm{IP}^{b}}$ & 2012126.04 & $300 \mathrm{~V}$ & $4 \times 895$ & 1.43 & +64 & 300 \\
\hline LTT1020 & 2012126.09 & $300 \mathrm{~V}$ & 15 & 1.01 & & \\
\hline $\mathrm{SN}^{2009 \mathrm{IP}^{b}}$ & 20121210.04 & $1200 \mathrm{R}$ & $4 \times 890$ & 1.53 & +68 & 200 \\
\hline LTT1020 & 20121210.09 & $1200 \mathrm{R}$ & 40 & 1.02 & & \\
\hline SN2009IP & 20121215.04 & $300 \mathrm{~V}$ & $4 \times 895$ & 1.73 & +73 & 150 \\
\hline LTT $1020^{c}$ & 20121215.09 & $300 \mathrm{~V}$ & 15 & 1.05 & & \\
\hline SN2009IP & 20121225.04 & $1200 \mathrm{R}$ & $4 \times 890$ & 2.10 & +83 & 50 \\
\hline LTT1020 ${ }^{c}$ & 20121225.09 & $1200 \mathrm{R}$ & 40 & 1.10 & & \\
\hline
\end{tabular}

are presented in Fig. 1. Line identifications indicated in the figure are taken from Fraser et al. (2013) and Margutti et al. (2014), where the spectroscopic evolution of SN 2009ip from this period can be found.

At +35 and $+42 \mathrm{~d} \mathrm{H} \alpha$ displays a distinct and asymmetric broad emission (full width at half-maximum, FWHM $\approx 10200 \mathrm{~km} \mathrm{~s}^{-1}$ ) with a narrow emission ( FWHM $\approx 300 \mathrm{~km} \mathrm{~s}^{-1}$ ) superimposed. A narrow absorption component can be seen with a minimum at $\sim-1500 \mathrm{~km} \mathrm{~s}^{-1}$ at +35 and $+42 \mathrm{~d}$. The absorption decreases in strength until it has disappeared by $+64 \mathrm{~d}$. At this stage, the shape of the broad emission has changed, becoming more reminiscent of $\mathrm{SNe}$ in the nebular phase. A broad absorption component with FWHM $\sim 10^{4} \mathrm{~km} \mathrm{~s}^{-1}$ is apparent at all epochs. Superimposed on this broad absorption are further narrow absorption notches at $-12300,-9700,-7300,-6000 \mathrm{~km} \mathrm{~s}^{-1}$, which we label A, B, C and $\mathrm{D}$, respectively. In the early epochs, the absorption notches $\mathrm{A}$, $\mathrm{B}$ and $\mathrm{C}$ are strongest, at $+64 \mathrm{~d}$ components $\mathrm{C}$ and $\mathrm{D}$ increase in strength before notch $D$ becomes weaker again at $+73 \mathrm{~d}$. These narrow components are more clearly visible in the $1200 \mathrm{R}$ observations. $\mathrm{H} \beta$ shows a broad absorption trough with a similar multicomponent velocity structure embedded, this is most prominent at $+42 \mathrm{~d}$. In contrast, $\mathrm{H} \beta$ does not show the same broad emission that $\mathrm{H} \alpha$ does.

A broad blend of $\mathrm{He}_{\mathrm{I}} \lambda 5876$ and $\mathrm{Na}$ I $\mathrm{D}$ in absorption is observed in the $300 \mathrm{~V}$ spectra. In all $1200 \mathrm{R}$ spectra, the Na I D doublet is resolved separately as narrow emission lines at 5889 and $5895 \AA$ (each with $\mathrm{FWHM} \approx 220 \mathrm{~km} \mathrm{~s}^{-1}$ ). These are emitted at the rest wavelength in the host galaxy. There is no sign of $\mathrm{He}_{\mathrm{I}} \lambda 5876$ in emission. Multiple absorption components are visible at $\sim 5640, \sim 5755$ and $\sim 5860$ $\AA$ at $+35 \mathrm{~d}$. We note that the feature around $5600-5900 \AA$ shows a similar structure to the $\mathrm{He}_{\mathrm{I}} 10830 \AA$ feature observed by Fraser et al. (2013). This similarity leads us to propose that the contribution of $\mathrm{Na}$ I D to the broad line feature is minor. The He I absorption is strongest at $\sim 5640 \AA$ with velocity $\sim-11800 \mathrm{~km} \mathrm{~s}^{-1}$, the other two components are observed at a velocity of -6200 and $-800 \mathrm{~km} \mathrm{~s}^{-1}$. The absorption components become stronger at $+42 \mathrm{~d}$ and a more complex velocity structure emerges, with further narrow absorption components appearing. The low-velocity $\left(<5000 \mathrm{~km} \mathrm{~s}^{-1}\right)$ absorption components increase in strength by $+64 \mathrm{~d}$, so that the feature appears to be composed of two separate absorption components at $\sim-4000$ and $\sim-12000 \mathrm{~km} \mathrm{~s}^{-1}$. Note that the higher velocity component may be Sc II $\lambda 5658$ in absorption, as has been identified in SN 1999em by Elmhamdi et al. (2003), Sc II $\lambda 5526$ is also seen in emission at +64 and $+73 \mathrm{~d}$. At +64 and $+73 \mathrm{~d}$, the narrow emission of $\mathrm{Na}$ I $\mathrm{D}$ is replaced by a broader emission resulting from the strengthening of the $\mathrm{He}_{\mathrm{I}} \lambda 5876$ emission line. This is supported by the increased strength of the $\mathrm{He}$ I $\lambda \lambda$ 5015, 6678 and $7065 \AA$ lines at those epochs.

The Ca II infrared (IR) triplet appears as three weak narrow emission lines with a potential underlying broad emission at +35 and $+42 \mathrm{~d}$, by the third epoch, the emission lines become more pronounced with associated blueward broad absorption troughs $\left(\sim-4000 \mathrm{~km} \mathrm{~s}^{-1}\right)$.

In general, the spectral evolution and line identifications discussed here are consistent with those presented in Fraser et al. (2013) and Margutti et al. (2014). The shoulder blueward to the narrow $\mathrm{H} \alpha$ component in the early spectra presented here and explained as a low-velocity $\mathrm{H} \alpha$ absorption at $\sim-1500 \mathrm{~km} \mathrm{~s}^{-1}$ is instead interpreted as an additional emission component with velocity $-3000 \mathrm{~km} \mathrm{~s}^{-1}$ by Fraser et al.

\section{ANALYSIS OF THE POLARIMETRY}

The polarization spectra of SN 2009ip from 2012 November 7 $(+35 \mathrm{~d})$ to the 2012 December 25 (+83 d) as observed with the $300 \mathrm{~V}$ and 1200R grisms are presented in Figs 2 and 3 below, respectively. The flux spectra are also shown in order to aid the identification of polarized signals associated with particular line features.

\subsection{Interstellar polarization}

Photons scattering from gas and dust grains in the interstellar and circumstellar material introduce a source of polarization in the spectrum that is not intrinsic to the transient. The removal of the ISP is therefore vital to the analysis so that we may understand the geometry of the event. 


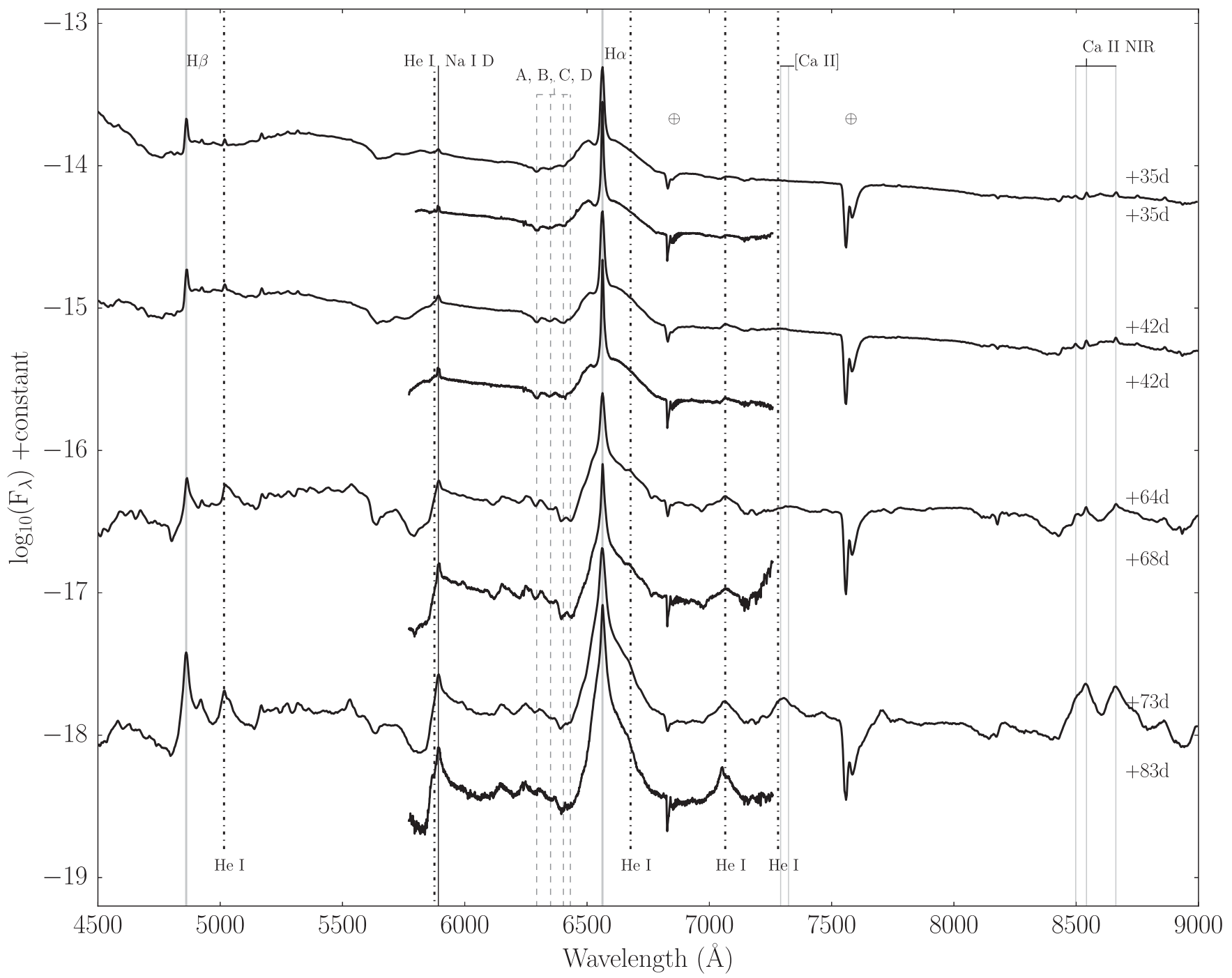

Figure 1. Flux spectra of SN 2009ip for the observations between 2012 November 7 (+35 d) and 2012 December 25 (+83 d) taken with the FORS2 instrument with the $300 \mathrm{~V}$ and 1200R grisms. Line identifications indicated are taken from Fraser et al. (2013) and Margutti et al. (2014), the vertical lines show the position of the rest wavelength for these lines. Also identified are the high-velocity narrow absorption 'notches' of H $\alpha$ : A, B, C and D. Telluric features are indicated by $\oplus$.

The foreground Galactic reddening for the Milky Way Galaxy (MW) along the line of sight to SN 2009ip is $E(B-V)=0.019$ mag (Schlegel, Finkbeiner \& Davis 1998). ${ }^{2}$ The ISP expected for the MW dust component is $<0.17$ per cent (Serkowski, Mathewson \& Ford 1975). The low polarization is corroborated by that of a standard star, HD 212146, 40' from the position of SN 2009ip, which has $p=0.08 \pm 0.04$ percent (Heiles 2000); however, the distance to this star is only $\sim 40 \mathrm{pc}$ (van Leeuwen 2007), such that it only provides a lower limit on the Galactic ISP arising along the line of sight.

The component of the ISP from the host galaxy is also expected to be small, as NGC 7259 is viewed face-on and SN 2009ip is positioned at the extreme edge of the optical disc at $4.3 \mathrm{kpc}$ (Fraser

\footnotetext{
${ }^{2}$ The NASA/IPAC Extragalactic Database (NED) is operated by the Jet Propulsion Laboratory, California Institute of Technology, under contract with the National Aeronautics and Space Administration. http://ned.ipac.caltech.edu
}

et al. 2013). The data (Figs 2 and 3) show no strong wavelength dependence in the continuum polarization, indicating that the ISP is small. A straight line fit with a least squares method to the Stokes $q$ and $u$ spectra at $+35 \mathrm{~d}$ with a first-order polynomial yielded a weak dependence on the wavelength of $\mathrm{d} u / \mathrm{d} \lambda=+1.5 \pm 1.9 \times 10^{-7} \AA^{-1}$ and $\mathrm{d} q / \mathrm{d} \lambda=+4.9 \pm 2.0 \times 10^{-7} \AA^{-1}$.

A value for the ISP was determined assuming that the emission of $\mathrm{H} \alpha$ was intrinsically unpolarized, such that the line polarization ( $q_{\text {line }}$ and $u_{\text {line }}$ ) at these wavelengths should reflect the ISP. Indeed, there is evidence of depolarization associated with the narrow emission line at all epochs and with the broad wings at +35 and $+42 \mathrm{~d}$. Two estimates of the line polarization were determined under different assumptions:

(i) $\mathrm{ISP}_{A}$ assumed that the broad emission of $\mathrm{H} \alpha$ under the narrow emission component was unpolarized;

(ii) $\mathrm{ISP}_{B}$ assumed that only the narrow emission component was unpolarized. 


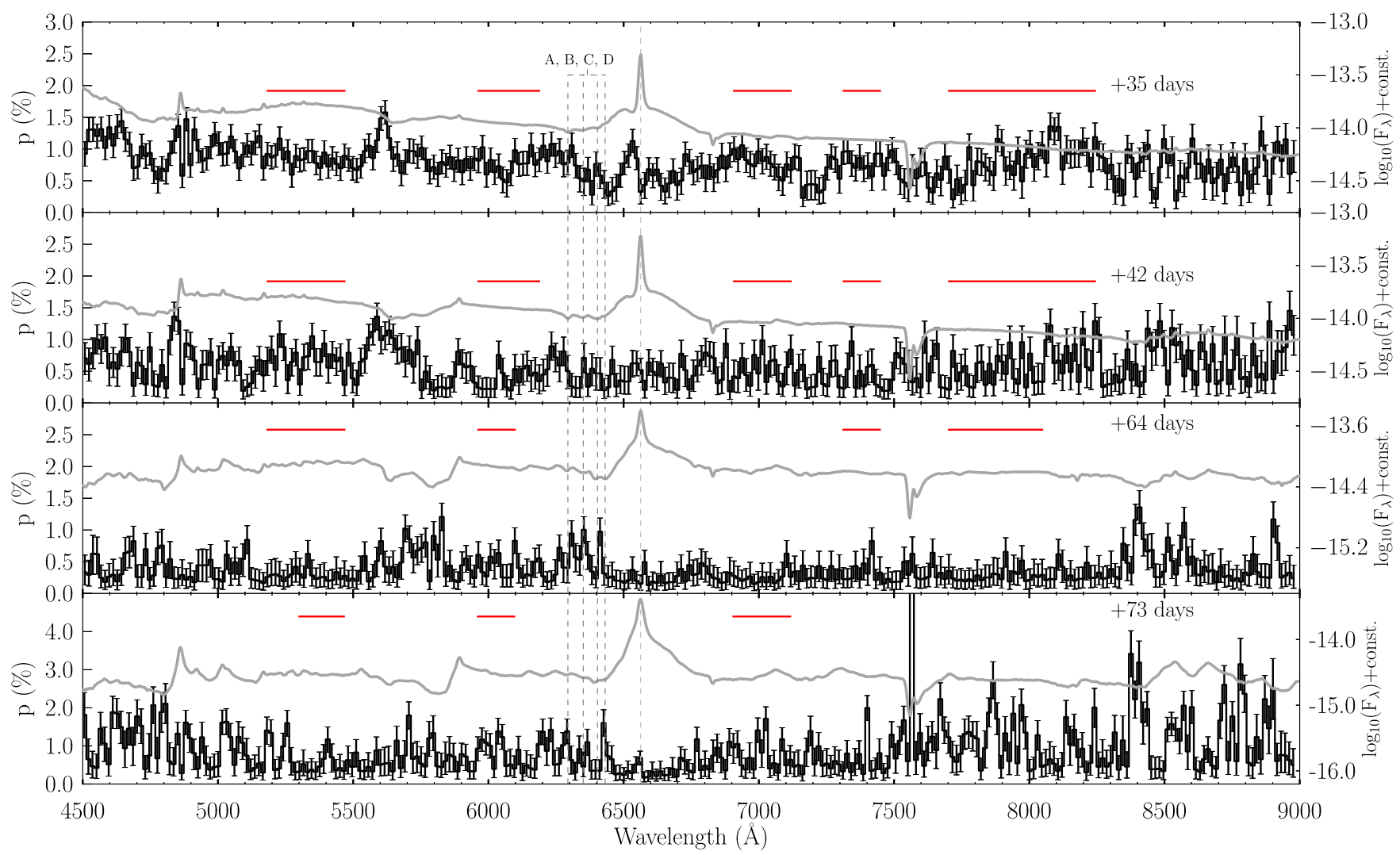

Figure 2. Spectropolarimetric data taken with the $300 \mathrm{~V}$ grism starting on 2012 November 7 and ending on 2012 December 15 . The degree of polarization as a function of wavelength is presented with the data binned to $15 \AA$ and corrected for the ISP. The flux spectra are in grey with units $10^{-14} \mathrm{erg} \mathrm{s}^{-1} \mathrm{~cm}^{-2} \AA^{-1}$ and are corrected for the recessional velocity of the host galaxy, $c z=1782 \pm 5 \mathrm{~km} \mathrm{~s}^{-1}$ (Meyer et al. 2004). The wavelength regions that were used to calculate the continuum polarization are shown by the red horizontal bars. Note the different scaling in each panel in flux and the degree of polarization.

The Stokes line polarization parameters, $q_{\text {line }}$ and $u_{\text {line }}$ are defined as follows;

$q_{\text {line }}=\frac{q_{\text {tot }} F_{\text {tot }}-q_{\text {base }} F_{\text {base }}}{F_{\text {line }}}$

$u_{\text {line }}=\frac{u_{\text {tot }} F_{\text {tot }}-u_{\text {base }} F_{\text {base }}}{F_{\text {line }}}$

These equations assume that, at any given wavelength, the observed total polarization is the sum of the line polarization (if any) and some base polarization that needs to be removed from the observed polarization to determine the line polarization. The flux of each component is denoted by $F$.

For $\mathrm{ISP}_{A}$, a flat continuum polarization was taken as the base polarization. The continuum polarization, $q_{\text {cont }}$ and $u_{\text {cont }}$, was determined as the weighted average of Stokes $q$ and $u$ in the wavelength regions indicated in Fig. 3. The continuum flux, $F_{\text {cont }}$ was measured from the bottom of the broad $\mathrm{H} \alpha$ wings. In this case, $q_{\text {base }}=q_{\text {cont }}$, $u_{\text {base }}=u_{\text {cont }}$ and $F_{\text {base }}=F_{\text {cont }}$ in the above equations.

For $\mathrm{ISP}_{B}$, the depolarization observed in Stokes $q$ and $u$ associated with the broad wings of $\mathrm{H} \alpha$ at +35 and $+42 \mathrm{~d}$, were approximated with a Gaussian profile. The line intensity was measured from the base of the narrow $\mathrm{H} \alpha$ P Cygni profile. To determine the line polarization in this case, the continuum polarization and the broad $\mathrm{H} \alpha$ component were accounted for such that

$q_{\text {base }}=q_{\text {cont }}+q_{\text {broad }}$ and similarly for $u_{\text {base }}$ and:

$F_{\text {base }}=F_{\text {cont }}+F_{\text {broad }}$

The $q_{\text {line }}$ and $u_{\text {line }}$ of a $5 \AA$ bin centred on the narrow $\mathrm{H} \alpha$ emission in the $1200 \mathrm{R}$ spectra were taken as the estimate of the ISP in each epoch. The higher spectral resolution 1200R data were chosen to avoid contamination from potential polarization in the broad wings of $\mathrm{H} \alpha$. The value adopted as the ISP was then taken as the inverse error weighted average of those from the first three of the four epochs of $1200 \mathrm{R}$ data sets presented. The $\mathrm{S} / \mathrm{N}$ of the polarization spectrum at $+83 \mathrm{~d}$ was considered too low to provide an accurate determination of the ISP. Averaging the measurements across multiple epochs makes the best use of the data, as the ISP should not vary with time.

$\mathrm{ISP}_{A}$ was found to be $q_{\mathrm{ISP}}=0.10 \pm 0.16$ percent and $u_{\mathrm{ISP}}$ $=-0.224 \pm 0.019$ percent, corresponding to $p_{\mathrm{ISP}}=0.18 \pm$ 0.13 percent and $\theta_{\text {ISP }}=146^{\circ} \pm 22^{\circ}$. This is consistent with the upper limit derived from the reddening above. Under the assumption that the broad emission was polarized, $\mathrm{ISP}_{B}$ was determined to be $q_{\mathrm{ISP}}=0.30 \pm 0.09$ per cent and $u_{\mathrm{ISP}}=-0.17 \pm 0.06$ per cent, corresponding to $p_{\mathrm{ISP}}=0.33 \pm 0.09$ per cent and $\theta_{\mathrm{ISP}}=164^{\circ} \pm$ $8^{\circ}$. The two estimates for the ISP agree to within their errors. The uncertainties on $q_{\mathrm{ISP}}$ and $u_{\mathrm{ISP}}$ are the standard deviation of the individual measurements from the three epochs around the final inverse error weighted average.

As $\mathrm{ISP}_{A}$ relied on fewer assumptions, this was the one adopted as the ISP. The ISP lies close to the origin on the $q-u$ plane, in the 


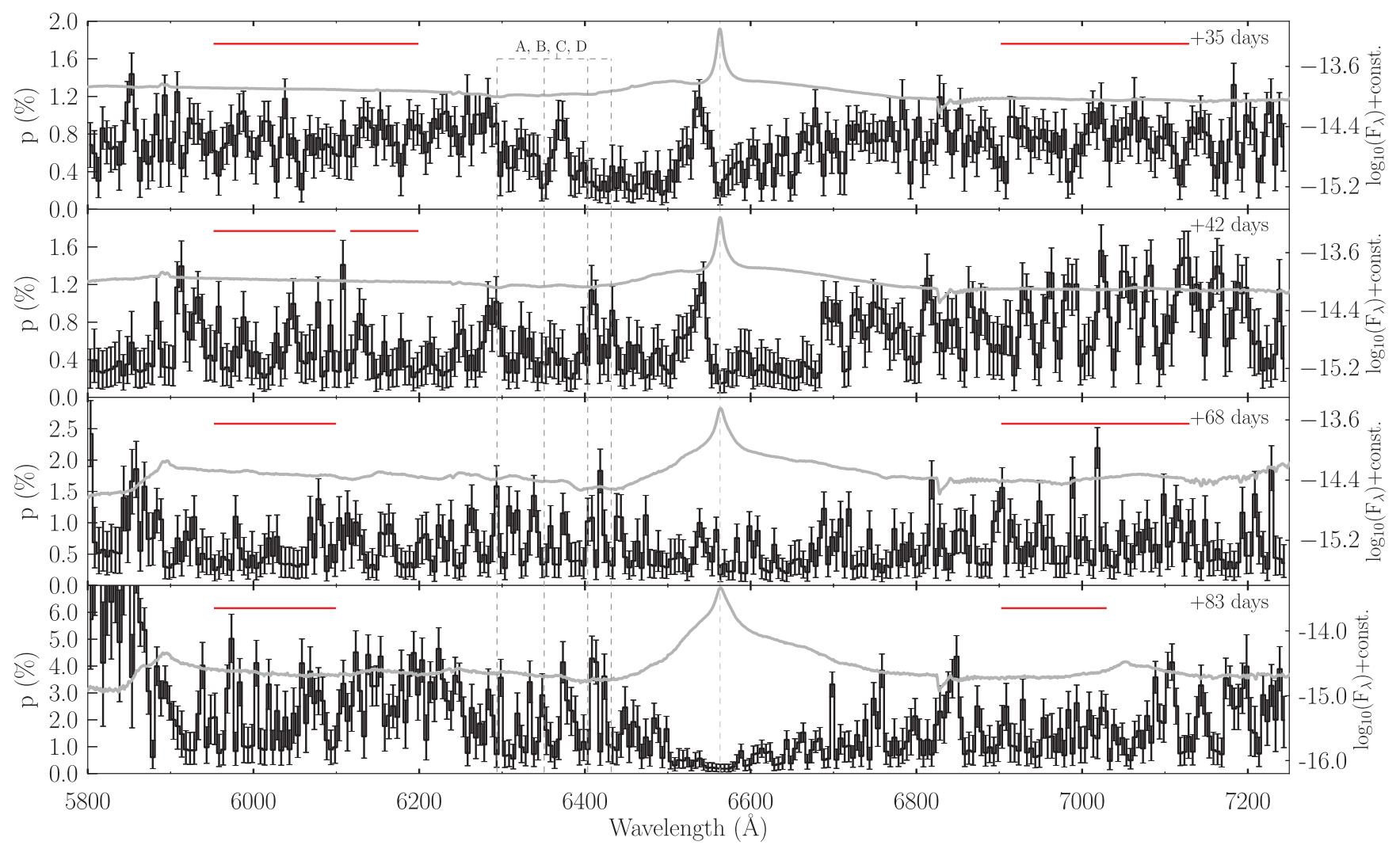

Figure 3. Same as Fig. 2, but for spectropolarimetric data taken with the 1200R grism starting on 2012 November 7 and ending on 2012 December 25.

positive $q$, negative $u$ quadrant, as marked in Fig. 4. As there is no discernible wavelength dependence in the observed continuum polarization at all epochs, we apply a single correction for the ISP to the entire wavelength range. $\mathrm{ISP}_{A}$ was subtracted from Stokes $q$ and $u$ and the uncertainties propagated by addition in quadrature, $p$ and $\theta$ were then recalculated.

\subsection{The intrinsic continuum polarization}

Upon removal of $\operatorname{ISP}_{A}$, the continuum polarization was determined by taking the inverse error weighted average of Stokes $q$ and $u$ in the wavelength regions specified in Figs 2 and 3. Wavelength regions where the polarization was approximately flat and where there were no obvious line features in the corresponding flux spectrum were chosen to determine the continuum polarization. The uncertainty of the Stokes $q$ and $u$ of the continuum corresponds to the standard deviation in the chosen regions. The degree and angle of polarization, $p$ and $\theta$, for the continuum were then calculated, with $p$ de-biased as described in Section 2. These are presented in Table 2.

The continuum is significantly polarized in the first epoch at $p$ $\sim 0.7$ per cent and $\theta \sim 45^{\circ}$. By the second epoch of $+42 \mathrm{~d}$, the degree of polarization has slightly decreased and is accompanied by a small rotation in the polarization angle. There is no significant change in the degree of polarization between +42 and $+64 \mathrm{~d}$, there is, however, a substantial change in the angle which undergoes a rotation of $\sim 60^{\circ}$. Following this, both the degree and angle of polarization remain approximately constant at the $1 \sigma$ level throughout the rest of the observations. The uncertainty in $p$ and $\theta$ increases in the later epochs due to the decreasing signal as the transient fades.
The rotation of the polarization angle by the third epoch is also clear from the evolution on the $q-u$ plane (Fig. 4). The large rotation occurs at the same time as a drop in the bolometric light curve as reported by Margutti et al. (2014). The change in the orientation of the polarization is possibly related to this event. The $I$-band light curve produced by Graham et al. (2014) is shown in Fig. 5 and also shows the drop in flux at $\sim+70 \mathrm{~d}$. The $I$-band light curve was chosen to display as the drop at $\sim+70 \mathrm{~d}$ is most obvious. The robustness of the observed rotation was tested by changing the determined $\mathrm{ISP}_{A}$ to its upper and lower $3 \sigma$ boundaries. The measured rotation was found to be partially dependent on the choice of ISP, with the rotation decreasing from $\sim 60^{\circ}$ to $\sim 20^{\circ}-40^{\circ}$. We consider it robust that the continuum polarization rotates, however the error introduced by the choice of ISP implies that the degree to which it rotates should be approached with caution.

The continuum polarization was calculated using wavelength ranges (as shown in Fig. 2) in which no major contribution from the polarization of line features was expected. In the later epochs, the He I $\lambda 7065$ emission line is visible in the 6900$7130 \AA$ region that is used to measure the continuum polarization at $+35,+42$ and $+73 \mathrm{~d}$, while the polarization spectrum is also noisy at $+73 \mathrm{~d}$. The validity of including this region in the measurement of the continuum polarization was determined through calculating the Stokes parameters excluding the region and comparing to the continuum polarization measured with the region around $7000 \AA$ included. The degree and angle of polarization are consistent within the uncertainties with each method. At $+73 \mathrm{~d}$, the continuum polarization is reduced by 0.05 per cent and by 0.01 per cent at +35 and $+42 \mathrm{~d}$ when the region is excluded. Exclusion of this region results in a 0.01 per cent increase in the uncertainty of the degree of 

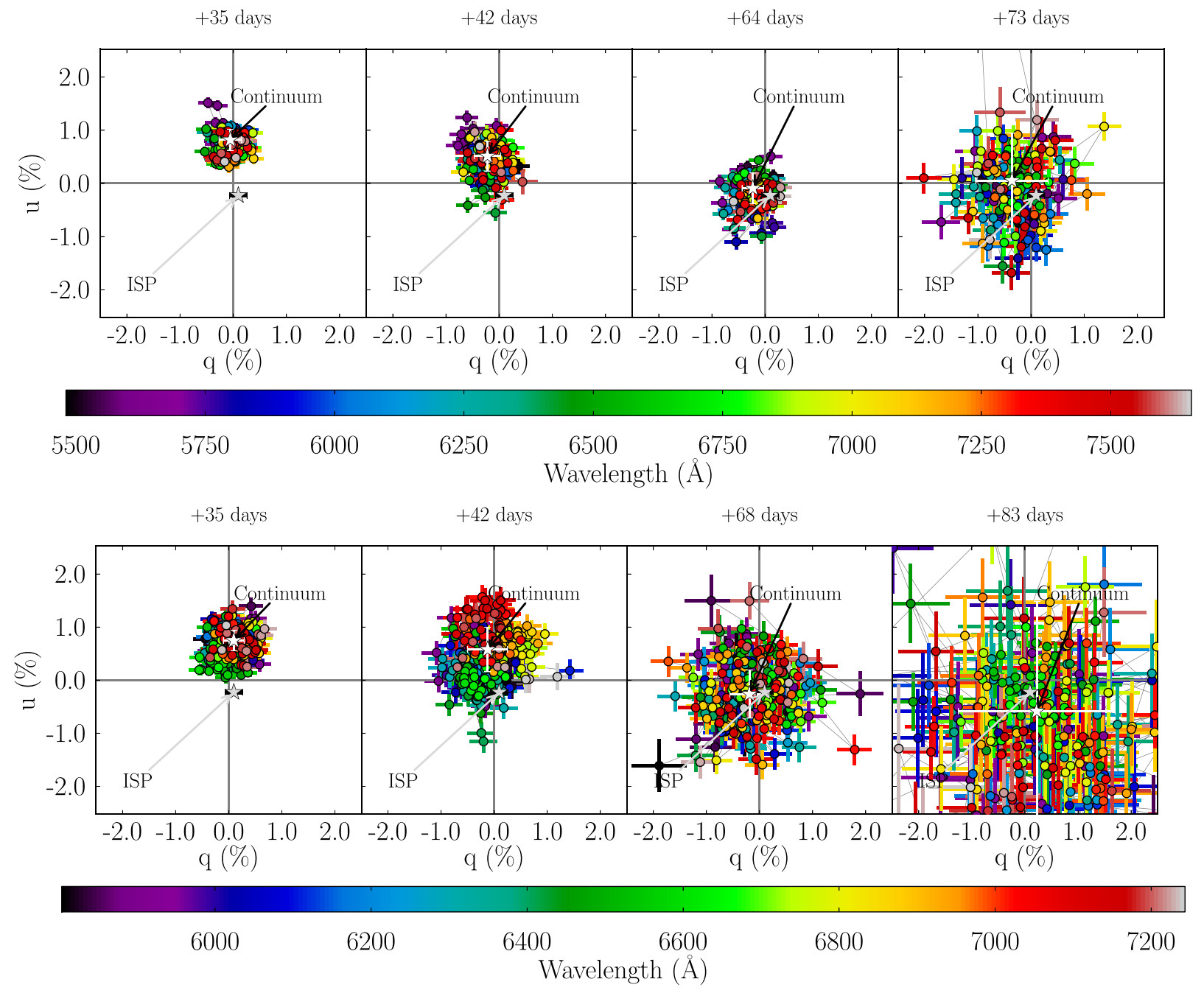

Figure 4. The evolution of SN 2009ip on the $q-u$ plane. The top panel contains the data from the 1200R observations (binned to $5 \AA$ ) and the bottom panel the observations taken with the $300 \mathrm{~V}$ grism (binned to $15 \AA$ ). The position of the continuum is marked by the white star with the $1 \sigma$ error bars. The data are corrected for the ISP shown as a grey star with black error bars.

Table 2. The ISP-corrected continuum polarization of SN 2009ip.

\begin{tabular}{lcc}
\hline Phase $(\mathrm{d})^{a}$ & $p$ (per cent) & $\theta\left(^{\circ}\right)$ \\
\hline & $300 \mathrm{~V}$ \\
+35 & $0.76 \pm 0.21$ & $47 \pm 8$ \\
+42 & $0.37 \pm 0.30$ & $57 \pm 21$ \\
+64 & $0.29 \pm 0.24$ & $100 \pm 15$ \\
+73 & $0.66 \pm 0.55$ & $89 \pm 21$ \\
& $1200 \mathrm{R}$ & \\
+35 & $0.69 \pm 0.20$ & $41 \pm 8$ \\
+42 & $0.33 \pm 0.26$ & $63 \pm 20$ \\
+68 & $0.48 \pm 0.39$ & $119 \pm 22$ \\
+83 & $0.78 \pm 1.07$ & $149 \pm 39$
\end{tabular}

Notes. ${ }^{a}$ Relative to UV-band maximum of the $2012 \mathrm{~b}$ rebrightening on 2012 October 3.

polarization at each epoch and a $1^{\circ}-5^{\circ}$ increase in the uncertainty of the polarization angle at +42 and $+73 \mathrm{~d}$. If the $\mathrm{He}$ I $\lambda 7065$ line is responsible for the noise in the polarization spectrum, it makes no significant contribution to the measurement of the continuum polarization.

\subsection{Intrinsic line polarization}

The flux spectra of SN 2009ip exhibit complex line features, with narrow and broad P Cygni profiles and absorption notches embedded at both low and high velocity. Similarly, the polarization spectra also show a complex structure, with some of the features common to multiple species. In the following subsections, we separate these features according to the breadth of the profile (narrow and broad features) and the velocity at which they appear, with respect to the rest wavelength of the line with which they are associated. The velocities, degree and angle of polarization of all the features summarized in the following subsections can be found in Table A1 in Appendix A.

\subsubsection{Broad polarized features: $H \alpha$}

In the flux spectrum, $\mathrm{H} \alpha$ is observed as a combination of a narrow P Cygni profile superimposed on a broad P Cygni profile. There is obvious depolarization associated with the broad emission $\left(\sim 10^{4} \mathrm{~km} \mathrm{~s}^{-1}\right)$ at +35 and $+42 \mathrm{~d}$, as can be seen in Figs 2 and 3 . No other species in the spectra shows the same broad emission or 


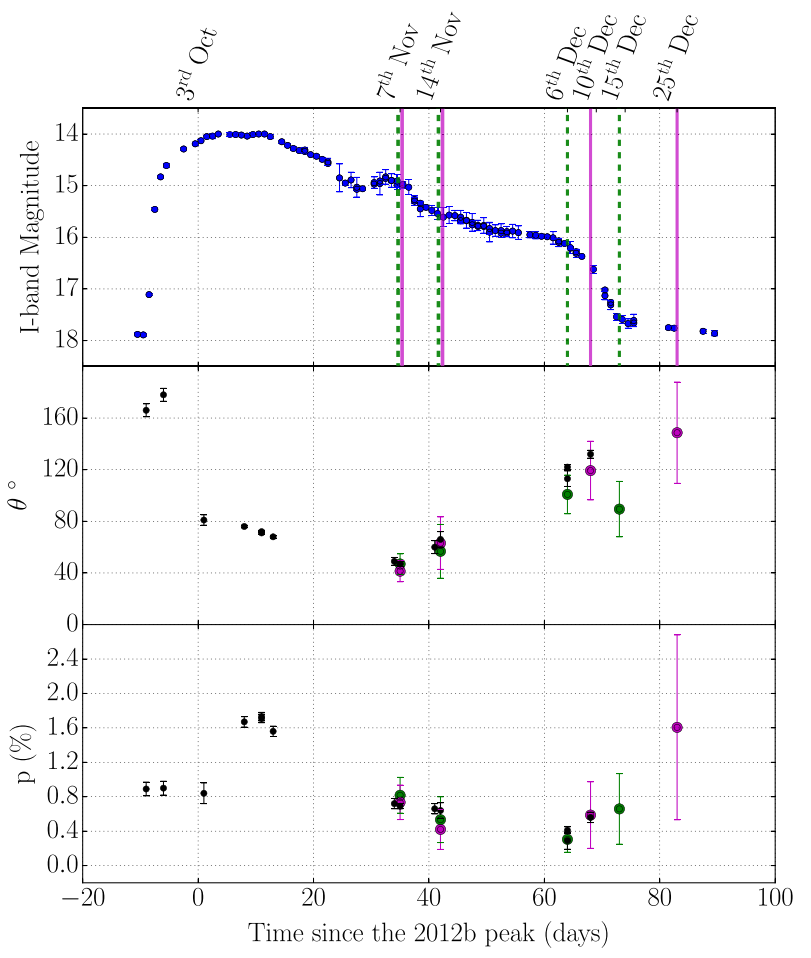

Figure 5. The evolution of the continuum polarization over the course of the $2012 \mathrm{~b}$ outburst. The top panel shows the $I$-band light curve using data presented by Graham et al. (2014) with the dates of the polarimetry observations overlaid with the dashed green lines for the $300 \mathrm{~V}$ data and solid magenta lines for the $1200 \mathrm{R}$ data. The polarization angle as a function of time is shown in the middle panel, followed by the polarization degree in the bottom panel. The smaller black markers represent the $V$-band polarization measurements as presented by Mauerhan et al. (2014).

depolarization at these epochs, which could be a consequence of the strength of the line.

For data taken at +35 and $+68 \mathrm{~d}$, the observed polarization, $p_{\text {obs }}$, of the broad $\mathrm{H} \alpha$ emission in 1200R was modelled with the intrinsic line polarization, $p_{\text {line }}$, as a variable. The model took the form of:

$p_{\text {obs }}=\frac{p_{\text {line }} F_{\text {line }}+p_{\text {cont }} F_{\text {cont }}}{F_{\text {obs }}}$

where the intensity of the continuum, $F_{\text {cont }}$ was fit with a secondorder polynomial. Two wavelength regions were chosen in order to determine whether the intrinsic polarization, $p_{\text {line }}$, of the broad emission was fixed or variable as a function of wavelength. These were $6600-6700 \AA$ (corresponding to $\sim 1700-6300 \mathrm{~km} \mathrm{~s}^{-1}$ ) for the core and $6700-6800 \AA\left(\sim 6300-10000 \mathrm{~km} \mathrm{~s}^{-1}\right)$ for the outer red wing of the line profile.

At $+35 \mathrm{~d}$, the observed polarization appears to be best fit with $p_{\text {line }} \leq 0.3$ per cent in the core. The $\chi_{v}^{2}$ was minimized (to a value of 1.68) for $p_{\text {line }}=0.2 \pm 0.1$ per cent. The improvement in $\chi_{v}^{2}$ for $p_{\text {line }}=0.2$ per cent over $p_{\text {line }}=0.0$ per cent is only fractional at 0.12 . The residuals of the fit of $p_{\text {obs }}$ to the observed $p$ are also evenly scattered for $p_{\text {line }} \leq 0.3$ per cent. The line polarization in the outer wings, however, is not as well constrained. The $\chi_{v}^{2}$ is minimized to a value of 1.38 by $p_{\text {line }}=0.5 \pm 0.1$ per cent. This corresponds to only a 0.043 improvement in the reduced chi-squared over either $p_{\text {line }}=$ 0.0 per cent or $p_{\text {line }}=0.9$ per cent. The data in this region appear to be equally well represented by either of these values. In this case, it is difficult to discern if the outer wings are more polarized than the core of the broad emission.

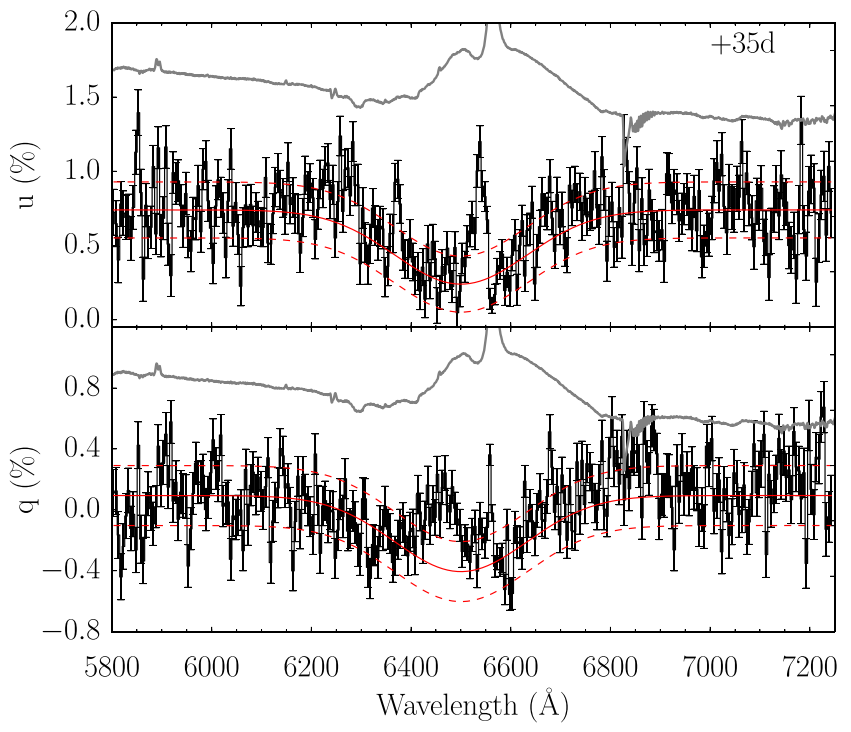

Figure 6. The fit to the broad $\mathrm{H} \alpha$ depolarization in $q$ and $u$ at $+35 \mathrm{~d}$ as observed with the 1200R grism. The top and bottom panels show $q$ and $u$, respectively, along with their Gaussian fit to the depolarization (the $\pm 1 \sigma$ fits are shown by the dashed red lines), used to isolate the polarization of the low-velocity narrow $\mathrm{H} \alpha$ line.

At $+68 \mathrm{~d}, \chi_{v}^{2}$ is minimized for $p_{\text {line }}=0.3 \pm 0.1$ per cent for both wavelength regions. This represents a 0.25 improvement in $\chi_{v}^{2}$ in the core over $p_{\text {line }}=0.0$ per cent, but only a 0.06 improvement for the outer wings. The data appear to be well represented by $p_{\text {line }} \leq$ 0.5 percent, with the increased scatter in the polarization meaning that it can be constrained no further.

The polarization blueward of the narrow emission is not well represented by the fits applied to the redward broad emission. This is also clear in Fig. 6, where the Stokes parameters across $\mathrm{H} \alpha$ at $+35 \mathrm{~d}$ are presented. The data show narrow (mostly in $u$ ) departures from the fit at $\sim 6370 \AA\left(v \sim-8820 \mathrm{~km} \mathrm{~s}^{-1}\right)$ and $\sim 6540 \AA$ $\left(v \sim-1000 \mathrm{~km} \mathrm{~s}^{-1}\right)$, these will be analysed in the following subsections. A broader $\left(\sim 5500 \mathrm{~km} \mathrm{~s}^{-1}\right)$ deviation from the fit is observed mostly in Stokes $q \sim 0.2-0.6$ per cent peaking at $\sim 6460 \AA$. This is indicative of polarization intrinsic to the broad P Cygni absorption.

The polarization angle of the broad component blueward of $\mathrm{H} \alpha$ was found using the $1200 \mathrm{R}$ spectra at $+35,+42$ and $+68 \mathrm{~d}$. An inverse error weighted average of $q_{\text {line }}$ and $u_{\text {line }}$ was calculated in regions avoiding the narrow polarization features associated with the absorption notches A, B, C and D. The feature between 6300 and $6500 \AA\left(\sim-12000\right.$ to $\left.-3000 \mathrm{~km} \mathrm{~s}^{-1}\right)$ was found to be polarized to the $p_{\text {line }} \sim 0.3-0.65$ per cent level from +35 to $+68 \mathrm{~d}$. The polarization angle was consistent at $\theta_{\text {line }} \sim 125^{\circ}$ in the first two epochs before rotating to $85^{\circ} \pm 42^{\circ}$, the large uncertainty on the latter value means that it is also consistent with the previous values to within $1 \sigma$.

\subsubsection{Low-velocity narrow polarization}

It can be seen from Fig. 7 that $\mathrm{H} \alpha, \mathrm{H} \beta$ and $\mathrm{He}$ I $\lambda 5876$ have complicated line profiles with multiple absorption components at both high and low velocity. In particular, they each show a narrow absorption component at $\sim-1500 \mathrm{~km} \mathrm{~s}^{-1}$. In the polarization spectra of $+35 \mathrm{~d}$, presented in Fig. 7, a large peak is observed associated with this component for $\mathrm{H} \alpha$ and $\mathrm{H} \beta$. This feature is also observed in the 1200R data for $\mathrm{H} \alpha$ and $\mathrm{He}_{\mathrm{I}} \lambda 5876$, as seen in Fig. 8. For 


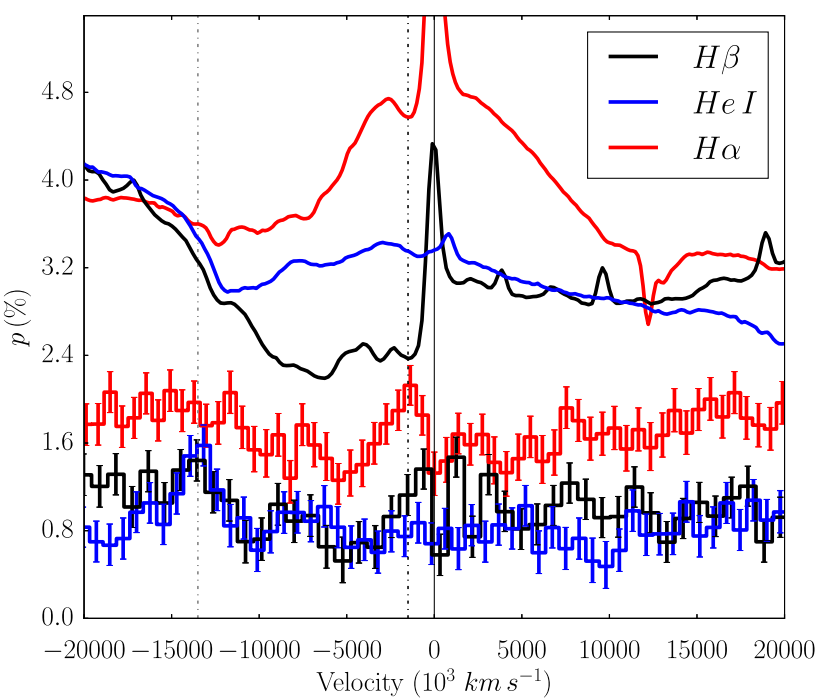

Figure 7. Line profiles and $300 \mathrm{~V}$ polarization spectra for $\mathrm{H} \alpha, \mathrm{H} \beta$ and $\mathrm{He} \mathrm{I}$ $\lambda 5876$ in velocity space at $+35 \mathrm{~d}$. The velocity of the absorption component (at absorption minimum) of the narrow P Cygni profile of $\mathrm{H} \alpha$ is indicated by the black dash-dotted vertical line. The grey dash-dotted line indicates the position of significant polarization observed for $\mathrm{H} \beta$ and $\mathrm{He}$ I $\lambda 5876$ at $\sim-14000 \mathrm{~km} \mathrm{~s}^{-1}$. Peaks in the polarization can also be seen at low velocities $\left(\sim-1000 \mathrm{~km} \mathrm{~s}^{-1}\right)$ for $\mathrm{H} \alpha$ and $\mathrm{H} \beta$. Note that the polarization spectrum across $\mathrm{H} \alpha$ (in red) has been offset by 1 per cent for clarity.

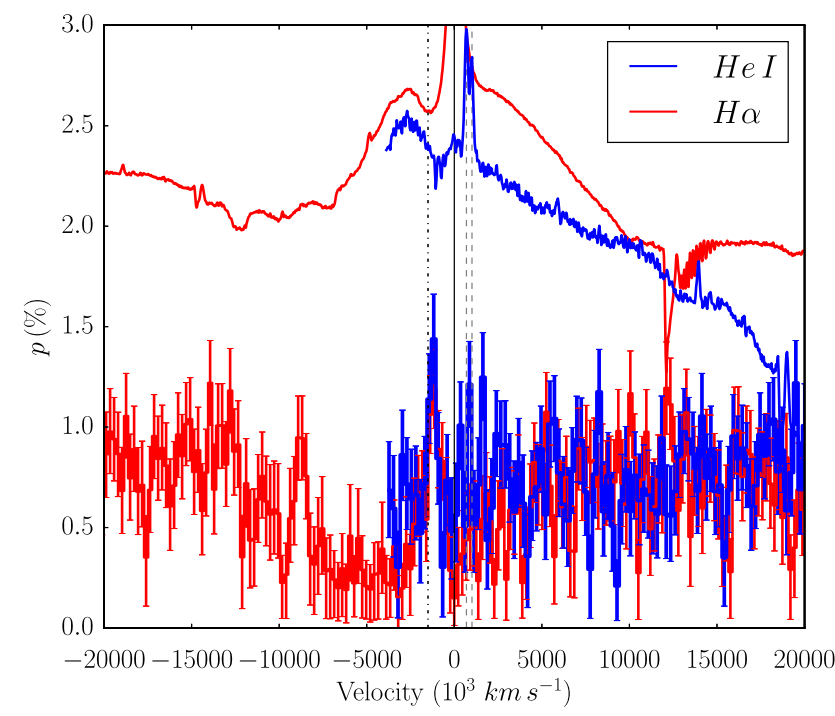

Figure 8. Same as Fig. 7 but for $\mathrm{H} \alpha$ and $\mathrm{He}$ I $\lambda 5876$ as observed with the $1200 \mathrm{R}$ grism at $+35 \mathrm{~d}$. The position of the Na I D emission lines are marked by the grey dashed lines. Again the velocity at absorption minimum for the narrow P Cygni profile of $\mathrm{H} \alpha$ is indicated by the black dash-dotted vertical line. A peak in the polarization is observed at $\sim-1000 \mathrm{~km} \mathrm{~s}^{-1}$ in both hydrogen and helium, implying a shared structure at low velocities for these line-forming regions. The He I profile is truncated as it fell at the blue edge of the wavelength range of the $1200 \mathrm{R}$ observations.

$\mathrm{H} \alpha$, the feature is evident on the $q-u$ plane in Fig. 9 at $+42 \mathrm{~d}$. It can be seen as a small number of data points in the upper left at low velocities $\left(\sim-1000 \mathrm{~km} \mathrm{~s}^{-1}\right)$ that protrude away from where the continuum is indicated. The $\mathrm{H} \alpha$ peak is visible until $+68 \mathrm{~d}$ in the $1200 \mathrm{R}$ data but is not polarized by more than $3 \sigma$ after $+35 \mathrm{~d}$ in the $300 \mathrm{~V}$ data. The difference in the strength of the feature be- tween the two data sets is largest at $+42 \mathrm{~d}$. Smoothing the $1200 \mathrm{R}$ data to the resolution of the $300 \mathrm{~V}$ grism and using the same bin size resolved this discrepancy. This highlights the need for highresolution spectropolarimetric observations for narrow line SNe. The degree of polarization decreases over time as the strength of the absorption in the flux spectrum decreases.

As the spectra contain a number of narrow features that are marginally significant we have employed a two-tailed $z$-test across our data (Sprinthall 2011). We only analyse those features where the two-tailed $z$-test gives a probability of more than $\sim 3 \sigma$ (having a probability less than 0.27 per cent) that the feature is not consistent with the continuum. The depolarization associated with the broad $\mathrm{H} \alpha$ component was accounted for when calculating the $z$-test statistic for the low-velocity narrow $\mathrm{H} \alpha$ signal and the highvelocity narrow 'notches'.

In order to isolate the line polarization of the low-velocity $\mathrm{H} \alpha$ feature, the depolarization of the broad $\mathrm{H} \alpha$ emission component was approximated with a Gaussian profile. This was then vector subtracted from the observed data. The fit to the 1200R Stokes $q$ and $u$ at $+35 \mathrm{~d}$ is shown in Fig. 6 . This was fit for both the $1200 \mathrm{R}$ and $300 \mathrm{~V}$ spectra at +35 and $+42 \mathrm{~d}$. For the later epochs, when there is no discernible depolarization, only the flat continuum polarization, as determined in Section 4.2, was subtracted.

In the $1200 \mathrm{R}$ spectrum of $+35 \mathrm{~d}$, the degree and angle of polarization of the low-velocity feature for $\mathrm{H} \alpha$ and $\mathrm{He}_{\mathrm{I}} \lambda 5876$ are consistent at $p_{\text {line }} \sim 1$ per cent and corresponding angle of $\theta_{\text {line }} \sim 33^{\circ}$. The signals display a striking similarity when compared in velocity, as seen in Fig. 8. For $\mathrm{H} \beta$, the degree of polarization, as revealed in the $300 \mathrm{~V}$ spectrum in Fig. 7, at this epoch is observed to be lower at $p_{\text {line }}=0.4 \pm 0.3$ per cent and $\theta_{\text {line }}=44 \pm 24^{\circ}$. The polarization of the same low-velocity $\mathrm{H} \alpha$ feature is also weaker in the $300 \mathrm{~V}$ data at $p_{\text {line }}=0.30 \pm 0.23$ per cent and the angle slightly rotated and with a larger uncertainty, $\theta_{\text {line }}=50^{\circ} \pm 15^{\circ}$. Similarly, the $\mathrm{He}_{\mathrm{I}}$ $\lambda 5876$ polarization is not observed in the $300 \mathrm{~V}$ spectrum taken on the same night (this is evident when Figs 7 and 8 are compared), demonstrating the loss of information when the spectra are binned to $15 \AA$.

At $+35 \mathrm{~d}$, the low-velocity polarized peaks of $\mathrm{H} \alpha$ and $\mathrm{H} \beta$ are redshifted with respect to the absorption line minimum observed in the flux spectrum (see Fig. 7). In the 1200R spectrum, this is by $350 \mathrm{~km} \mathrm{~s}^{-1}$ for $\mathrm{H} \alpha$, where for $\mathrm{He}_{\text {I }} \lambda 5876$ the polarized peak is blueshifted from the absorption minimum. It is possible that blending with $\mathrm{NaI}$ D has smeared out the total flux line profile. Given the similarity in the polarized signals in velocity space, it would appear that $\mathrm{Na}$ I D does not significantly contribute to the polarized line profile.

The $\mathrm{H} \alpha$ signal remains polarized through to $+68 \mathrm{~d}$ where it is detected at $2.8 \sigma$ (according to the $z$-test) and exhibits no significant change in the polarization angle. In contrast, the low-velocity He I $\lambda 5876$ feature is not observed after $+35 \mathrm{~d}$. The $\mathrm{H} \beta$ signal, conversely, increases in degree of polarization to $p_{\text {line }}=1.1 \pm$ 0.3 per cent and undergoes a rotation to $98^{\circ} \pm 9^{\circ}$ before dissipating by $+64 \mathrm{~d}$. The similarity in the polarization of $\mathrm{H} \alpha, \mathrm{H} \beta$ and $\mathrm{He} \mathrm{I}$ $\lambda 5876$ at low velocities indicates that hydrogen and helium share an asymmetric slowly moving $\left(\sim-1100 \mathrm{~km} \mathrm{~s}^{-1}\right)$ line-forming region.

\subsubsection{Polarization at intermediate and high velocities}

Multiple species show peaks in the polarization at intermediate and high velocities associated with the observed absorption components (see Fig. 7). Similarly to the low-velocity feature discussed in the 


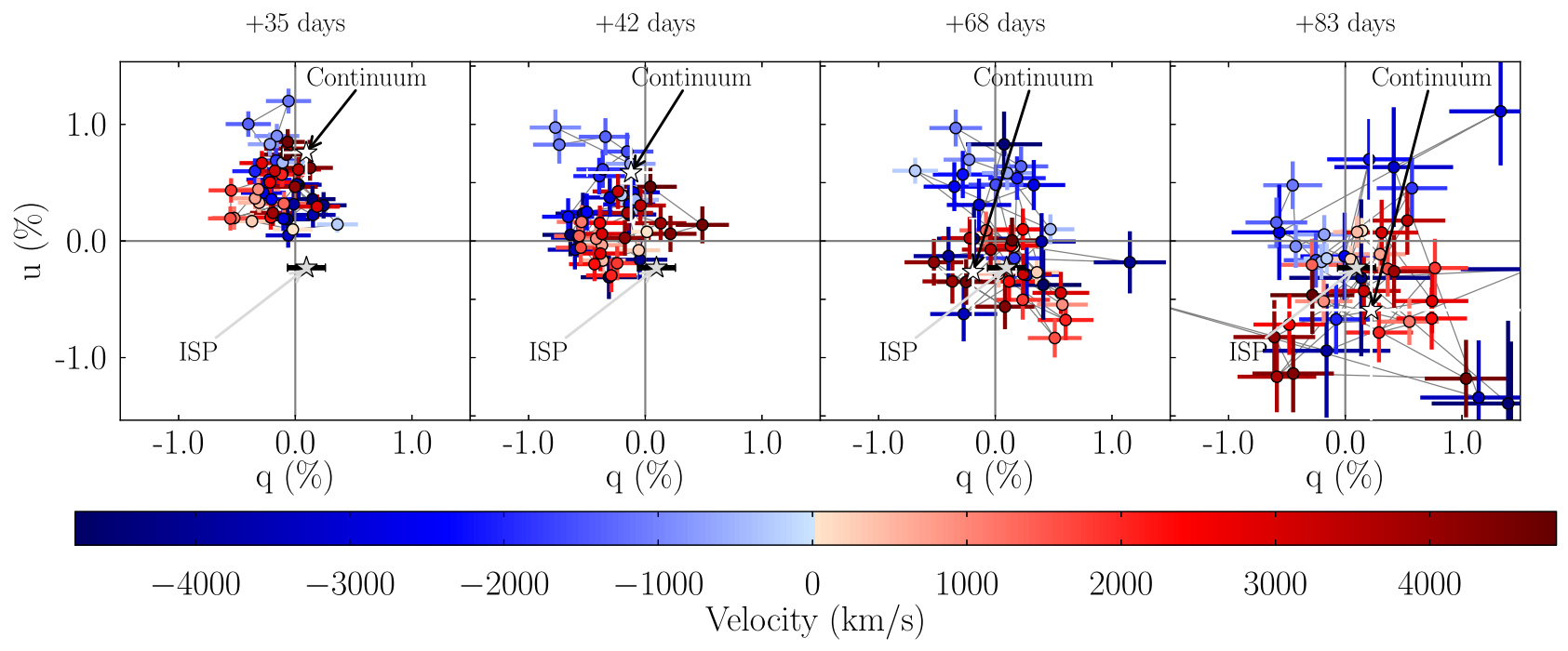

Figure 9. The evolution of $\mathrm{H} \alpha$ on the $q-u$ plane as observed with the $1200 \mathrm{R}$ grism in place using $5 \AA$ A binning. The position of the continuum is marked by the white star with the $1 \sigma$ error bars. The position of the ISP is indicated by the grey star. Stokes $q$ and $u$ have been corrected for the ISP in these plots.

previous subsection, some polarized features have corresponding signals associated with another line or species, indicating that they may arise in similar line-forming regions. This, however, is not the case for all detected line polarization. Further information of the polarization of features summarized here can be found in Table A1 in Appendix A.

At $+35 \mathrm{~d}, \mathrm{He} \mathrm{I} \lambda 5876$ and $\mathrm{H} \beta$ display peaks at $\sim-14000 \mathrm{~km} \mathrm{~s}^{-1}$ with $p_{\text {line }} \sim 0.5-0.7$ per cent and consistent polarization angle of $\theta_{\text {line }} \sim 55^{\circ}$. Fig. 7 shows that the profiles are also similarly shaped in velocity space. Both polarization peaks are also blueshifted from the nearest absorption minimum by $\sim 2000 \mathrm{~km} \mathrm{~s}^{-1}$. Similarly, at $+64 \mathrm{~d}, \mathrm{H} \alpha$ and $\mathrm{He}$ I $\lambda 5876$ share a peak of strength $p_{\text {line }}=0.7 \pm 0.3$ per cent and angle of $\theta_{\text {line }} \sim 120^{\circ}$ at $-9800 \mathrm{~km} \mathrm{~s}^{-1}$. At $+73 \mathrm{~d}, \mathrm{H} \beta$ and $\mathrm{He}$ I $\lambda 5876$ again show comparable polarization at $-6350 \mathrm{~km} \mathrm{~s}^{-1}$ at a similar position in the sky. In flux, the velocity structure of $\mathrm{H} \alpha, \mathrm{H} \beta$ and $\mathrm{He}$ I $\lambda 5876$ are similar as shown in Fig. 7 ; the structure in polarization corroborates this. The data imply that at high velocities as well as low, hydrogen and helium share the same asymmetrical line-forming regions.

At $+42 \mathrm{~d}$, there is significant polarization at the absorption minimum of the $\mathrm{H} \alpha$ feature labelled 'notch C' $\left(v_{\text {line }}=-7300 \mathrm{~km} \mathrm{~s}^{-1}\right)$ in the $1200 \mathrm{R}$ data. Its line polarization was determined in the same way as that of the low-velocity $\mathrm{H} \alpha$ feature discussed above. It is observed with $p_{\text {line }}=1.0 \pm 0.4$ per cent and $\theta_{\text {line }}=141^{\circ} \pm 12^{\circ}$. There is no significant polarization associated with other lines that could be interpreted as arising from the same line-forming region as this feature at this epoch.

In the later epochs of +64 and $+73 \mathrm{~d}$, there are several polarized peaks in the range of $p_{\text {line }} \sim 0.6-1.4$ per cent for $\mathrm{H} \alpha, \mathrm{H} \beta$ and $\mathrm{He} \mathrm{I}$ $\lambda 5876$ that have no obvious corresponding feature associated with other lines. The polarization angles of these features mostly cluster around the same region of the sky at $\sim 120^{\circ}$. At $+64 \mathrm{~d}$, three features are detected in the $300 \mathrm{~V}$ data associated with $\mathrm{H} \alpha$ at -7000 to $-12150 \mathrm{~km} \mathrm{~s}^{-1}$ with polarization at the $0.6-0.8$ per cent level. In contrast, no significant polarization is detected in this velocity range in the $1200 \mathrm{R}$ spectrum of four days later at $+68 \mathrm{~d}$. It is possible that these features have dissipated in that time, however Fig. 3 shows that there are some rises in the polarization in this range. The uncertainty on the continuum polarization is higher at $+68 \mathrm{~d}$ than in the $300 \mathrm{~V}$ spectrum of $+64 \mathrm{~d}$; the larger uncertainty could mean that these features are present but not detected as being significantly different from the continuum polarization at $+68 \mathrm{~d}$.

The Ca II IR triplet only becomes polarized at $+64 \mathrm{~d}$ in the $300 \mathrm{~V}$ data (see Fig. 2), associated with the strengthening of the emission lines at this epoch (Fig. 1). Strong line polarization is observed in the two distinct absorption troughs. The velocities of the polarized features were determined by using the nearest redward emission as the rest wavelength. Note that these may be inaccurate due to ambiguity in which of the components of the triplet is responsible for the feature. At $+64 \mathrm{~d}$, the maximum observed polarization associated with the Ca II IR triplet is $p_{\text {line }}=1.1 \pm 0.4$ per cent, the components in both absorption troughs have similar consistent polarization angles of $\sim 100^{\circ}$. At $+73 \mathrm{~d}$, both of these features strengthen. The feature in the trough of the $8542 \AA$ line (at $\lambda=8375 \AA$ ) is most strongly polarized at $p_{\text {line }}=3.4 \pm 0.9$ per cent, the polarization of the $8662 \AA$ line, on the other hand, increases to $1.7 \pm 0.9$ per cent. It is probable that the blended nature of the emission has diluted the polarization from the $8662 \AA$ line, such that it is not as highly polarized as the $8542 \AA$ line.

\subsection{Evolution of SN 2009ip on the polar diagram}

Fig. 10 shows the evolution of the polarization angle for different lines as a function of velocity. The polarization angles of each species, as described above, are presented as arcs centred on $\theta_{\text {line }}$, and the length of the arc represents the $\pm 1 \sigma$ uncertainty.

The $1200 \mathrm{R}$ data are particularly useful for studying the absorption component of the low-velocity narrow $\mathrm{H} \alpha$ P Cygni profile. The polarization angle of this feature does not display much temporal evolution, lying at approximately $45^{\circ}$ during all of the observations. It is closely aligned with the low-velocity $\mathrm{He}_{\mathrm{I}}$ feature and the continuum polarization angle at +35 and $+42 \mathrm{~d}$. The broad $\mathrm{H} \alpha \mathrm{P}$ Cygni absorption line is polarized at $\sim 90^{\circ}$ to the narrow feature. The high-velocity narrow notch labelled $\mathrm{C}(+42 \mathrm{~d})$ is highly misaligned with the narrow $\mathrm{P}$ Cygni profile and the continuum 

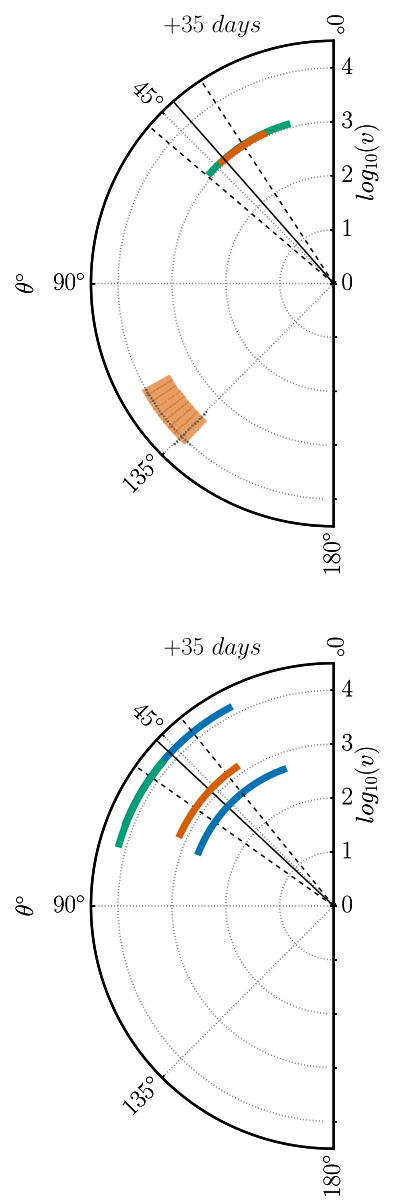
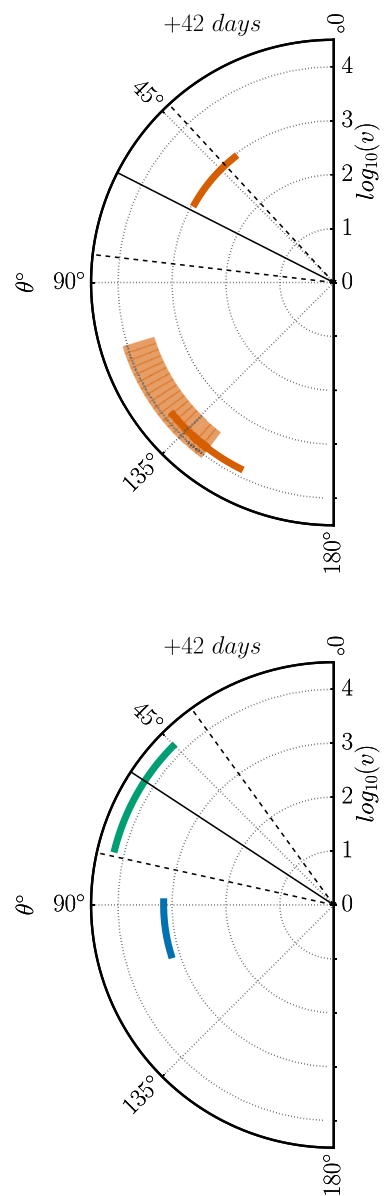
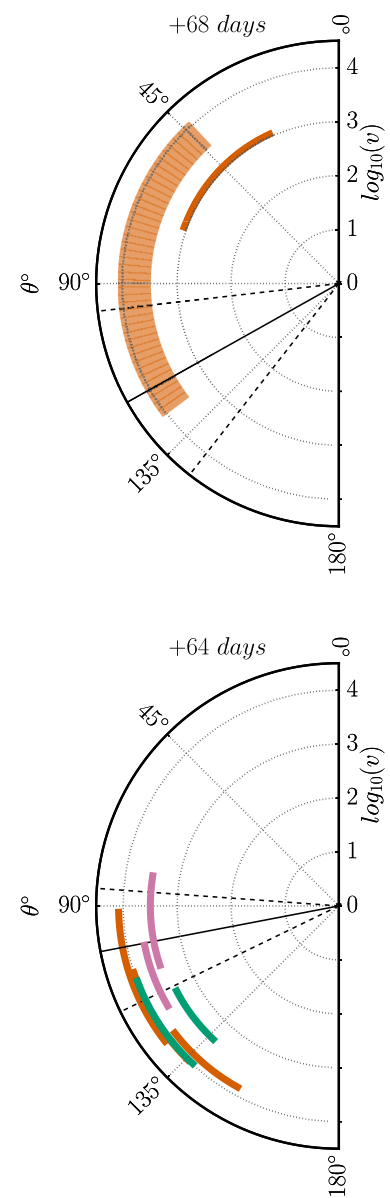
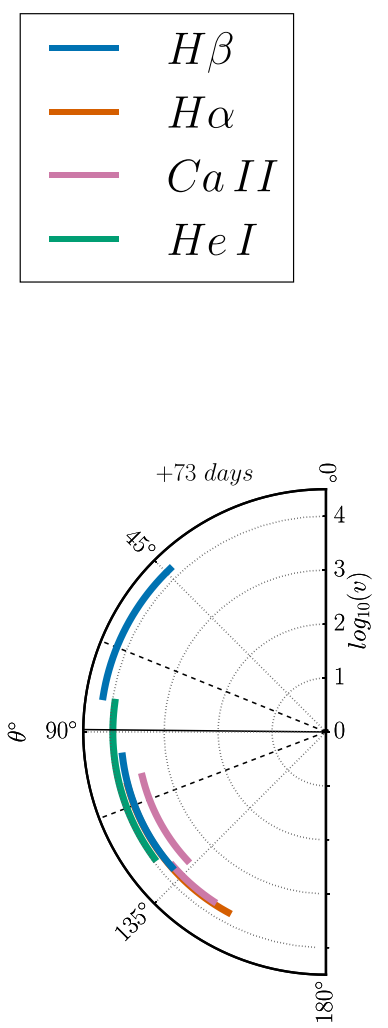

Figure 10. Top panel: $1200 \mathrm{R}$ polar plots of the polarization angle of the $\mathrm{H} \alpha$ and $\mathrm{He} \mathrm{I} \lambda 5876$ absorption lines, the velocity in $\log _{10}\left(v \mathrm{~km} \mathrm{~s}^{-1}\right)$ is indicated radially. Bottom panel: $300 \mathrm{~V}$ polar plots showing the angle of $\mathrm{H} \alpha, \mathrm{H} \beta, \mathrm{He}$ I $\lambda 5876$ and $\mathrm{Ca}$ II IR triplet absorption polarization. The polarization angle of the broad $\mathrm{H} \alpha \mathrm{P}$ Cygni absorption is shown by the slightly transparent orange wedge. The radial extent of the wedge represents that the measurement is the average of the polarization angle between -12000 and $-3000 \mathrm{~km} \mathrm{~s}^{-1}$. The continuum is marked with the black lines with the $1 \sigma$ uncertainty indicated by the dashed lines.

at this epoch; instead it is aligned with the broad $\mathrm{H} \alpha$ P Cygni absorption component.

The $300 \mathrm{~V}$ data show that the polarization angles of all species and the continuum at $+35 \mathrm{~d}$ are concentrated around $45^{\circ}$, including hydrogen and helium travelling at $\sim-14000 \mathrm{~km} \mathrm{~s}^{-1}$. At $+42 \mathrm{~d}$, the continuum undergoes a slight counterclockwise rotation, which is observed in both the $300 \mathrm{~V}$ and $1200 \mathrm{R}$ data. The low-velocity $\mathrm{H} \beta$ polarized feature rotates to $\sim 90^{\circ}$, where the high-velocity helium polarization remains at $60^{\circ}$. At $+64 \mathrm{~d}$, the continuum polarization angle has rotated further to $\sim 100^{\circ}$, the material with $v>4000 \mathrm{~km} \mathrm{~s}^{-1}$ is also clustered around the same position, between $90^{\circ}$ and $150^{\circ}$. This remains the case at $+73 \mathrm{~d}$ except for one component of $\mathrm{H} \beta$ at $-15250 \mathrm{~km} \mathrm{~s}^{-1}$ that is polarized at $\sim 60^{\circ}$. The continuum polarization shows a slight clockwise rotation at $+73 \mathrm{~d}$, whereas its previous evolution was in a counterclockwise fashion. As mentioned in Section 4.2, the evolution of the continuum polarization angle is sensitive to the choice of the ISP.

\section{DISCUSSION}

\subsection{The geometry of interacting transients}

The polarimetric observations of SN 2009ip add to the small sample of only three previous interacting transients studied with this technique, SN 1997eg, SN 1998S and SN 2010jl (Hoffman et al. 2008; Leonard et al. 2000; Wang et al. 2001; Patat et al. 2011; Bauer et al. 2012). All three SNe showed significant continuum polarization in the $\sim 2-3$ per cent range. Developing an interpretation of the geometry of Type IIn SNe can be a difficult task considering the ambiguity surrounding the question of what fraction of the observed polarization is due to electron scattering in an asymmetric photosphere as opposed to asymmetries in the interaction region. Given that SN 1997eg, SN 1998S and SN 2010jl were interactiondominated transients, the high degrees of continuum polarization have in some cases been interpreted as indicating significant departures from spherical symmetry in the circumstellar material. For 
these objects, the geometry of the CSM has been inferred to be disclike. At late times (>100 d), both SN 1997eg and SN 1998S display broad double or triple peaked Balmer lines, with equatorial disc-like enhancement considered as the explanation. The elevated polarization in the blue wings of $\mathrm{H} \alpha$ of SN 1997eg led Hoffman et al. to the conclusion that the CSM was disc-like for that SN. Andrews et al. (2011) found that a disc or torus-like geometry was required for SN 2010j1 in order to explain the low optical depth along the line of sight in conjunction with the high IR excess observed. X-ray observations also indicate an aspherical CSM formation for SN 2010jl (Chandra et al. 2015).

Despite the small sample size a somewhat consistent picture of these objects is emerging. The highly aspherical, toroidal or disc-like CSM observed in other Type IIn SNe is also invoked by Levesque et al. (2014), Fraser et al. (2013), Mauerhan et al. (2013), Ofek et al. (2013) and Smith et al. (2014) to explain various observations of SN 2009ip. Levesque et al. used this geometry to explain the observed low ratio of $\mathrm{I}(\mathrm{H} \alpha) / \mathrm{I}(\mathrm{H} \beta)$, which required an extraordinarily high density for the CSM. Ofek et al. (2013) found that the mass-loss rate derived from the $\mathrm{H} \alpha$ luminosity was an order of magnitude higher than the mass loss derived from the X-ray and bolometric luminosity. This required that the narrow $\mathrm{H} \alpha$ component be formed at radii larger than the shock radius in a spherical CSM or that the surrounding material was aspherical in geometry. It is apparent that an aspherical structure surrounding SN 2009ip is the natural explanation for many of the otherwise difficult-to-reconcile observations.

Polarimetric observations of SN 2009ip at around peak maximum light also indicate that the CSM is highly aspherical. Mauerhan et al. (2014) published multiepoch spectropolarimetric data from 2012 September 21 (during the 2012a re-brightening) to 2012 December $10(+68 \mathrm{~d})$, and included data that are presented here. The $V$-band polarization measurements made by Mauerhan et al. (2014) are shown in Fig. 5 for reference. Mauerhan et al. noted a rotation in the continuum polarization angle of $90^{\circ}$ between the $2012 \mathrm{a}$ observations (taken at $\sim-12 \mathrm{~d}$ with respect to the $2012 \mathrm{~b}$ October 3 maximum) and observations taken around the peak of the $2012 b$ explosion (at $\sim+11 \mathrm{~d}$ ), from which they inferred orthogonal axes of symmetry for the structures of the $2012 \mathrm{a}$ and $2012 \mathrm{~b}$ events. They suggested that the polarization angle of the continuum during the 2012 a event $\left(\sim 166^{\circ}\right)$ represented the axis of symmetry of the ejecta from an SN explosion occurring on 2012 July 24 and that the onset of $2012 \mathrm{~b}$ marked the beginning of interaction of that ejecta with an orthogonal disc-like circumstellar component. The orientation of the latter was inferred from the continuum polarization angle of $\sim 72^{\circ}$ measured around the $2012 \mathrm{~b}$ peak.

The observations presented here are discussed in the following subsections within the context of the previous observations of SN 2009ip and interacting transients in general.

\subsection{Origin of the broad $\mathrm{H} \alpha$ profile and continuum polarization}

In Type IIn $\mathrm{SNe}$, the broad component of $\mathrm{H} \alpha$ may either arise in the reprocessing of photons due to electron-scattering in the shocked dense CSM (Chugai 2001) or in the reverse-shocked ejecta. In the first case, electron-scattering would produce broad Lorentzian wings with photons upscattered to high velocities through multiple scattering events. If the line-forming region is asymmetric, the upscattered photons in the outer wings of $\mathrm{H} \alpha$ would be significantly more polarized than the core. The increased polarization is because the photons making up the outer wings are more likely to have undergone polarizing scattering incidents (in order to reach the higher velocities) than those arising in the core of the line, some of which may have escaped without electron scattering.

In Section 4.3.1, we analysed the polarization signal of the red wing of the broad P Cygni component of $\mathrm{H} \alpha$. At both +35 and $+68 \mathrm{~d}$, there is little evidence to support an increase in the line polarization in the outer wings compared to the core. This suggests that there is minimal reprocessing of $\mathrm{H} \alpha$ by a shell of electrons and therefore makes formation of this component in an external optically thick CSM unlikely.

The lack of reprocessing is not unexpected as the broad emission at this time dramatically differs from the Lorentzian profile observed at the peak of the light curve, instead it resembles a P Cygni profile (see Fig. 8 and Mauerhan et al. 2013, 2014; Fraser et al. 2013; Margutti et al. 2014). The observed $\sim 90^{\circ}$ separation in polarization angle between the narrow and broad $\mathrm{H} \alpha$ absorption lines (Fig. 10) would also indicate that the two components do not arise in the same line-forming region.

Around the time of our first observation the continuum polarization angle is observed to rotate significantly from earlier measurements (see Fig. 5 and Mauerhan et al. 2014). The evidence suggests that the interaction had begun to fade before our observations (when the ejecta travelling at $\sim 8000 \mathrm{~km} \mathrm{~s}^{-1}$ would be $\sim 6.7 \times 10^{10} \mathrm{~km}$ from the centre of the explosion) and that the broad lines and photosphere now reside in the ejecta rather than the interaction as is thought to be the case at maximum light.

Note that the beginning of our observations is concurrent with a 'bump' in the light curve at $\sim+35 \mathrm{~d}$ (Fig. 5). There is no consensus on the source of this fluctuation (or the others present in the light curve). Suggestions for the origin of the bumps include heating from a surviving progenitor (Martin et al. 2015), interactions of shells ejected from a binary system (Soker \& Kashi 2013; Kashi et al. 2013), the temporary re-brightening of the ejecta photosphere (Mauerhan et al. 2014) or interaction with an inhomogenous CSM (Graham et al. 2014). Martin et al. (2015) proposed that they could arise from the reheating of the inner rim of the disc-like CSM that Levesque et al. (2014) suggested was responsible for the $2012 \mathrm{~b}$ outburst. See Martin et al. (2015) for an in-depth analysis of the fluctuations in the light curve. The observations presented above suggest that, regardless of the source of the 'bump', the continuum polarization is no longer dominated by electron-scattering in the interaction at the time of our observations.

\subsection{Origin of the low-velocity $\mathrm{H} \alpha$ polarization}

The observed polarization of the low-velocity $\left(\sim-1000 \mathrm{~km} \mathrm{~s}^{-1}\right)$ $\mathrm{H} \alpha$ absorption may be used qualitatively to constrain the structure of the CSM. The relatively weak absorption strength coupled with the significant polarization indicates that the CSM obscures a small and highly aspherical region of the photosphere from +35 to $+68 \mathrm{~d}$. This may point to the equatorial enhancement or disc-like structure surrounding the progenitor that was suggested in previous studies (Fraser et al. 2013; Mauerhan et al. 2013, 2014; Ofek et al. 2013; Levesque et al. 2014; Smith et al. 2014). The observed absorption depth and polarimetric signatures of such a CSM are dependent upon a number of parameters, such as the orientation of the plane of the non-spherical CSM, the extent perpendicular to that plane $z$ (the thickness of the disc), and the inclination with respect to the line of sight $i$. These parameters may then be used to rule out various configurations. For example, at certain inclinations a disclike geometry may cover large portions of the photosphere and induce very strong absorption and polarization if the photosphere is 
not uniformly obscured, at larger inclinations, however, it may not obscure the photosphere at all.

In an effort to understand the geometrical configurations that could produce the low-velocity $\mathrm{H} \alpha$ polarization, a model of the photosphere and line-forming region was constructed following the prescription of Wang, Baade \& Patat (2007) and Maund et al. (2010), and described in detail in Reilly et al. (2016). The model simulated $1 \times 10^{7}$ photons across a limb-darkened 2D elliptical photosphere and tested the polarization induced by blocking it with simple lineforming regions. To simulate the polarization of the $2 \mathrm{D}$ photosphere, each photon was assigned either a random polarization angle or one tangential to the ellipse at the position of the photon. This depended on the distance of the photon's origin from the centre of the photosphere, with photons arising closer to the limb less likely to be randomly polarized. The probability of non-random polarization was set such that for a given axis ratio, the resulting continuum polarization reproduced the polarization expected for an oblate ellipsoid as specified by the models of Höflich (1991).

The polarization observed in the $1200 \mathrm{R}$ data at +35 and $+68 \mathrm{~d}$ was simulated. As the narrow $\mathrm{H} \alpha$ absorption is strongly polarized but weakly absorbing, line-forming regions that would induce a strong polarization while only absorbing a small fraction of the photospheric photons were chosen. These were assumed to completely absorb all photons originating from the area of the photosphere obscured by the line-forming region. The total normalized Stokes vectors of the escaping photons were calculated. The continuum, line and total observed polarizations were calculated in the same manner as for the observational data here. The fraction of photons absorbed by the line-forming region was calculated and compared to that absorbed in one $5 \AA$ bin in the observed 1200R flux spectrum.

The observed continuum polarization was reproduced at +35 and $+68 d$ with an axis ratio of $0.88(0.90$ at $+68 d)$, as expected from Höflich (1991), implying deviations from spherical symmetry on the 10-15 per cent level for the photosphere for these epochs. Rotating the ellipse such that the long axis of the photosphere was at $135^{\circ}\left(30^{\circ}\right.$ for $\left.+68 \mathrm{~d}\right)$ from the $y$-axis (North) reproduced the continuum polarization angle of $41^{\circ} \pm 8^{\circ}\left(119^{\circ} \pm 22^{\circ}\right.$ for $\left.+68 \mathrm{~d}\right)$. The line-forming regions were first tested for the data at $+35 \mathrm{~d}$ before successful models were then tested again given the continuum polarization at $+68 \mathrm{~d}$. Two line-forming regions were found to successfully approximate the line and total observed polarization and the absorption depth of the narrow $\mathrm{H} \alpha$ profile at $+35 \mathrm{~d}$. These represent two possible scenarios for the CSM: a disc-like structure observed edge-on with the photosphere oblate with respect to the plane of the disc or alternatively a similar structure inclined with respect to the observer and the photosphere prolate to the plane of the disc. These scenarios are shown in Fig. 11 and the observed and simulated data can be seen in Fig. 12 and Table B1 in Appendix B.

\subsubsection{Edge-on disc-like CSM scenario}

A thin rectangular line-forming region, similar to that projected by an edge-on disc-like or equatorial enhancement, was found to approximately reproduce the observed properties of the low-velocity $\mathrm{H} \alpha$ feature. The line-forming region, as shown in the top panel of Fig. 11, had a long axis that aligned with that of the photosphere. The absorption depth and degree of polarization were found to be sensitive to the length of the short axis (corresponding to the extent perpendicular to the plane of the CSM or the thickness of the disc). In order to reproduce the polarimetric properties, a short axis with an extent of $11 \pm 1$ per cent that of the long axis of the photo-

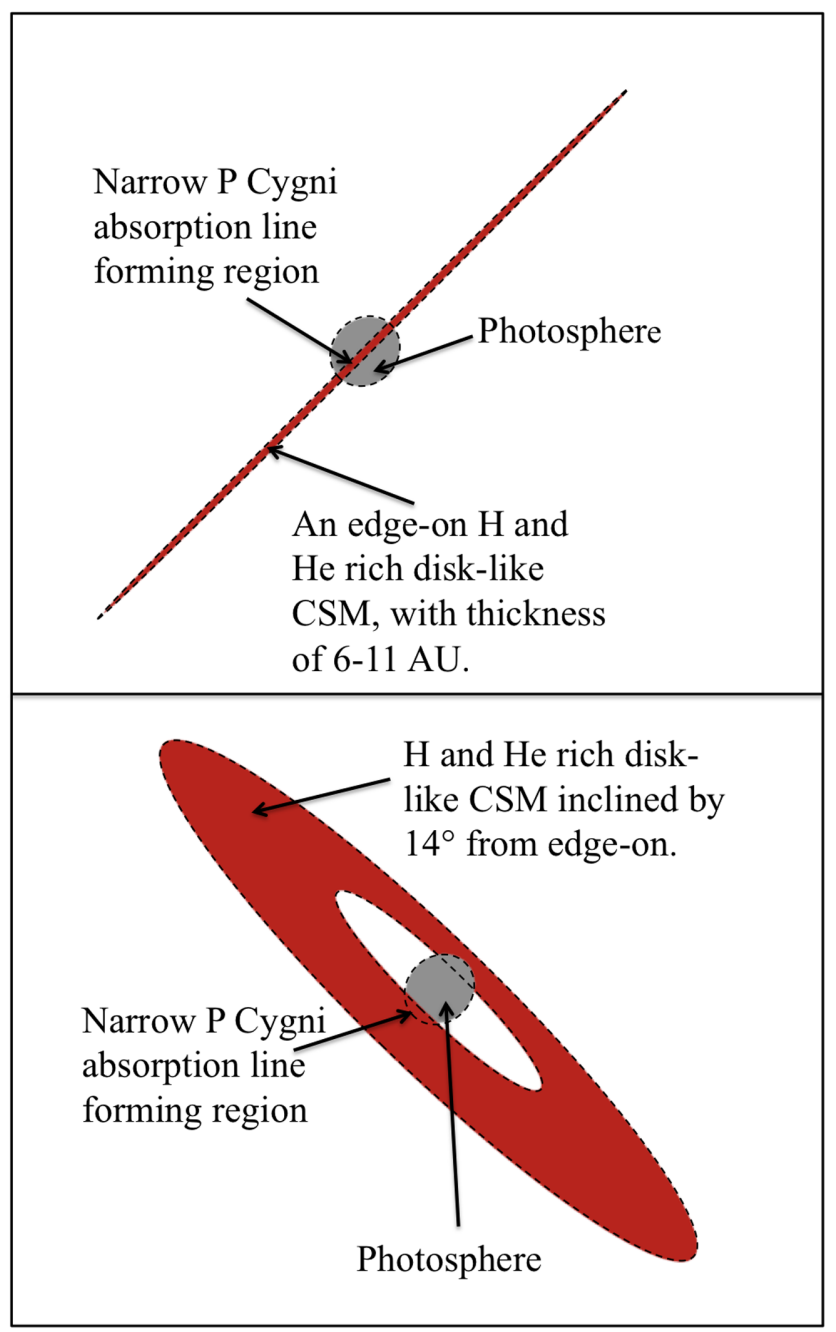

Figure 11. Schematics of the successful geometries that reproduced the polarization of the continuum and the low-velocity $\mathrm{H} \alpha$ feature at $+35 \mathrm{~d}$. The top panel shows the scenario where the observer views a disc-like CSM edge-on. In this case, the photosphere is oblate with respect to the long axis of the CSM. The bottom panel shows the scenario where this geometry is inclined out of the line of sight by $14^{\circ}$, where the photosphere appears to be prolate with respect to the long axis of the CSM.

sphere was required. This corresponded to $11 \pm 1 \mathrm{AU}\left(\sim 1.65 \times 10^{9}\right.$ $\mathrm{km}$ ) assuming a photospheric radius of $1.5 \times 10^{10} \mathrm{~km}$ at $+35 \mathrm{~d}$ (Margutti et al. 2014). This was found to overestimate the absorption depth by $\sim 10$ per cent. A thinner line-forming region of $6 \mathrm{AU}$ $\left(\sim 0.90 \times 10^{9} \mathrm{~km}\right)$ could reproduce the fraction of absorbed photons but was inconsistent with the observed degree of polarization. The first configuration was also found to successfully reproduce the polarimetry at $+68 \mathrm{~d}$, the absorption depth however was larger than that observed at $+35 \mathrm{~d}$, which is inconsistent with the observations, considering that the low-velocity feature is not observed in absorption in the latter epoch (Table B1).

The orientation of this CSM parallel to the major axis of the photosphere has the implication that the continuum polarization at $+35 \mathrm{~d}$ arises in a pseudo-photosphere created by electron-scattering at the interface between the CSM and the ejecta. Furthermore, the disappearance of the narrow $\mathrm{H} \alpha$ absorption and the polarization at $\sim+70 \mathrm{~d}$ imply that the ejecta have swept up or passed the outer edge of the CSM by this epoch. Considering an explosion epoch of 2012 


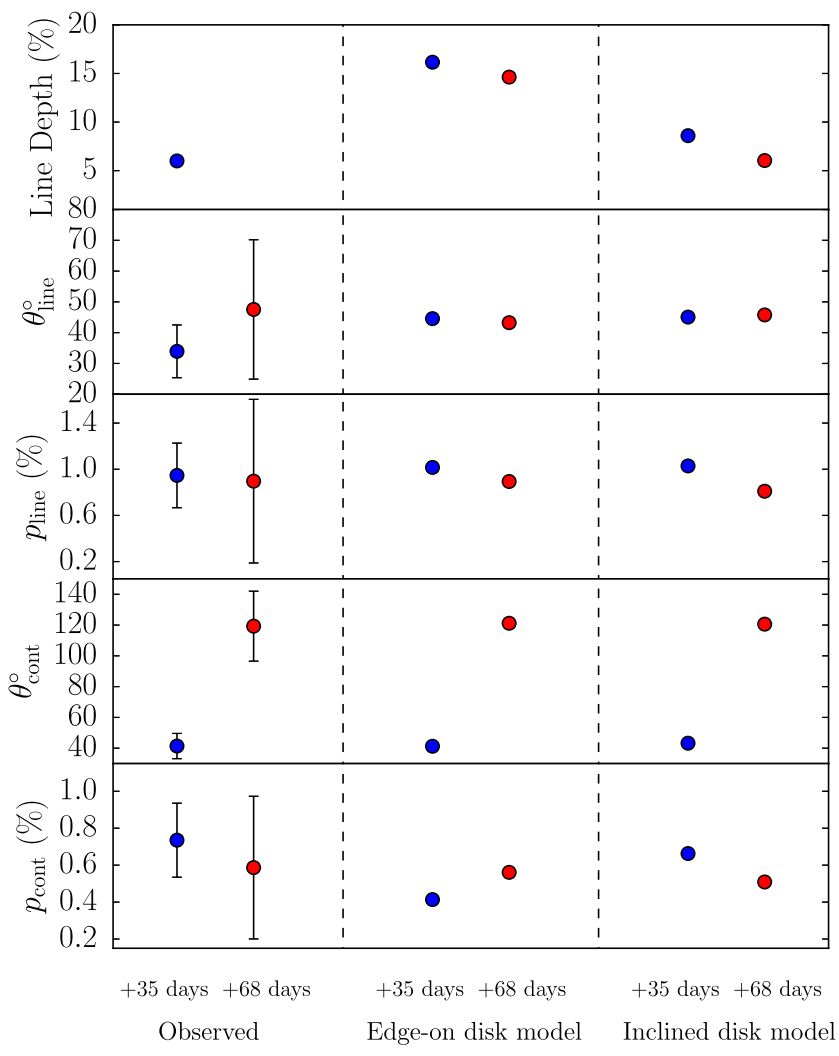

Figure 12. The continuum and low-velocity $\mathrm{H} \alpha$ polarization properties as observed in the $1200 \mathrm{R}$ data and as predicted by the Monte Carlo simulations of the edge-on and inclined disc-like CSM models of SN 2009ip (Fig. 11) at +35 and $+68 \mathrm{~d}$.

July 24 (the onset of 2012a) and a velocity of $-8000 \mathrm{~km} \mathrm{~s}^{-1}$ for the bulk of the ejecta, the outer edge of the disc can then be constrained to $10.2 \times 10^{10} \mathrm{~km}$.

\subsubsection{Inclined disc-like CSM scenario}

The observed properties were also reproduced by absorbing photons in the limb of the long axis of the photosphere (as seen in the bottom panel of Fig. 11); similar to how a disc-like CSM that is slightly inclined with respect to the observer would appear. In this case, the photosphere is prolate with respect to the plane of the CSM. This was performed by taking a disc-like CSM with a circular inner radius and inclining it incrementally. Assuming a bulk velocity flow of $-8000 \mathrm{~km} \mathrm{~s}^{-1}$ for the ejecta, an explosion epoch corresponding to the onset of 2012a (2012 July 24) and the beginning of 2012b (2012 September 23) as marking the onset of the interaction of the ejecta with the CSM, the position of the inner edge of the CSM was estimated to be $4.2 \times 10^{10} \mathrm{~km}$. Inclining this structure by $14^{\circ} \pm 2^{\circ}$ (given a photospheric radius of $1.5 \times 10^{10}$ $\mathrm{km}$, Margutti et al. 2014) with respect to the observer approximately simulated the observed polarization at both +35 and $+68 \mathrm{~d}$. The absorption depth was also slightly closer to that observed at $+35 \mathrm{~d}$ and showed a larger decrease for $+68 \mathrm{~d}$, which is more consistent with the observations than that simulated with the edge-on model CSM above. The simulated Stokes parameters were sensitive to changes of a few degrees in the inclination, however uncertainty in the position of the inner radius of the disc results in an increase in the uncertainty of the inferred inclination of the CSM disc.
By comparing the blackbody radius inferred by Margutti et al. (2014) to the projected extent of the inner radius of the simulated CSM, we determined that the disc would begin to block the photosphere and produce an absorption feature just prior to the $2012 \mathrm{~b}$ maximum. The absorption feature would then disappear at $\sim+70 \mathrm{~d}$ as the photosphere retreated. Indeed, the polarized feature is observed to disappear between +68 and $+83 \mathrm{~d}$. In this scenario, the low-velocity $\mathrm{H} \alpha$ polarization is produced by the same material that interacts with the ejecta at earlier times to power the $2012 \mathrm{~b}$ maximum.

\subsubsection{A model geometry for the CSM}

The alignment of the photosphere with the plane of the CSM in the edge-on disc scenario at $+35 \mathrm{~d}$ may imply that the continuum photons are polarized by electron-scattering in the interaction region (a pseudo-photosphere). This is difficult to reconcile with the inferred lack of reprocessing of photons discussed in Section 5.2. Similarly, the orientation of such a pseudo-photosphere in these observations at $\sim 40^{\circ}$ is inconsistent with the continuum polarization angle observed during the $2012 \mathrm{~b}$ maximum of $\sim 70^{\circ}$ (Mauerhan et al. 2014; their $V$-band polarization observations are shown in Fig. 5 for reference). Mauerhan et al. (2014) suggested that the continuum at that time arose in the interaction between a disc-like CSM and the ejecta. In the inclined-disc scenario, the production of the continuum polarization in a pseudo-photosphere is unnecessary as the photosphere lies in a plane perpendicular to that of the CSM. This suggests that the photosphere instead arises in the ejecta as is also implied by the lack of reprocessed photons at these epochs. It is also difficult to understand the presence of an edge-on disc-like $\mathrm{CSM}$ out to $10.2 \times 10^{10} \mathrm{~km}$, given that the transient had become ejecta-dominated before our observations, implying an outer radius of $\sim 6.7 \times 10^{10} \mathrm{~km}$ for the CSM.

Predictions of the emergence and disappearance of the absorption in the inclined-disc scenario match the observations and explain the lack of narrow absorption during the $2012 \mathrm{a}$ outburst. Under the assumption that the observed CSM was formed before the explosion, an edge-on disc would be expected to produce absorption during the early outburst which is not observed. This discrepancy may be avoided under certain formation scenarios which suggest that the observed geometry was created during or after the 2012a event. It is, however, difficult to avoid the special circumstances that would be required to observe a thin disc edge-on. While in the reference frame of the star such a structure is just as likely to form at any orientation with respect to the star, placing that structure along the line of sight to Earth would be statistically remarkable.

The continuum polarization and low-velocity $\mathrm{H} \alpha$ absorption depth at both +35 and $+68 \mathrm{~d}$ were also slightly better reproduced with the inclined-disc model (Fig. 12 and Table B1). For the reasons above, we favour the inclined scenario simulated here and shown in the bottom panel of Fig. 11. Given the shared absorption and polarized components of hydrogen and helium at low velocity (Figs 7, 8 and 10), the helium feature likely shares this inclined-disc geometry.

We note that the successful simulation of the data with two distinct geometries highlights that this simple model suffers from degeneracies and since there are two scenarios that replicate the data well, there may be more. Similarly, the scenarios determined here may not reproduce the polarimetry under models that include the density of the CSM and full radiative transfer. 


\subsection{Polarimetric implications for the geometry}

From our spectropolarimetric data, we have identified two distinct phases in the evolution of SN 2009ip: phase 1 - the geometry inferred from the first epoch of our observations and phase $2-$ the geometry inferred at around $\sim+70 \mathrm{~d}$.

The first phase occurs at $+35 \mathrm{~d}$. At this epoch strong line polarization reveals a hydrogen- and helium-rich circumstellar disc, or equatorial ring, inclined by $\sim 14^{\circ}$, such that it blocks a portion of the photosphere. The continuum polarization indicates that the ejecta was oriented prolate with respect to the plane of the disc at this time (bottom panel of Fig. 11). Using the models of Höflich (1991), the continuum polarization of $\sim 0.8$ per cent at $+35 \mathrm{~d}$ implies an axial ratio of $\sim 0.85$ (or 15 per cent asymmetry) for an ellipsoidal photosphere and indicates a bipolar structure for the SN ejecta. We observe that the polarized components of high-velocity He I $\lambda 5876$ and $\mathrm{H} \beta$ align with those at low velocity (Fig. 10), indicating that this material obscures the region of the photosphere as the CSM disc.

In Section 5.2, we discussed that the change in the $\mathrm{H} \alpha$ line profile, in conjunction with the rotation of the continuum polarization at around $+35 \mathrm{~d}$ compared to earlier observations, signified that the outburst was no longer interaction-dominated. The persistence of narrow lines at these epochs implies that interaction is ongoing but does not dominate the continuum light. Viewing the ejecta while there is ongoing interaction is possible in the light of our polarimetric results, which favour a highly aspherical inclined CSM. This would allow the ejecta to expand freely along the axis orthogonal to the CSM, while the CSM would also not block the entirety of the photosphere from the observer. This scheme has previously been suggested by Smith et al. (2014) and Fraser et al. (2013) to explain the high velocities observed in SN 2009ip and by Mauerhan et al. (2014) to explain their observation of polarized He I/Na I D that was blue shifted with respect to the absorption minimum in the flux spectrum.

The orthogonality of the broad and narrow P Cygni absorption of $\mathrm{H} \alpha$ (Fig. 10) may indicate that the fast-moving ejecta lie above and below the equatorial disc. In this way, the presence of the disc prevents it from blocking the same region of the photosphere that the disc obscures. The observed polarization, therefore, does not necessarily imply an intrinsic deviation from spherical symmetry for the broad $\mathrm{H} \alpha$-forming region of the ejecta. The continuum polarization at $+35 \mathrm{~d}$ however, indicates that the photosphere is elongated with the long axis approximately perpendicular to the plane of the CSM, suggesting asymmetry in the fast-moving ejecta.

The aspherical ejecta could be the result of inherent asymmetry in the explosion mechanism (with the major axis orthogonal to that of previous eruptions which formed the CSM) or due to shaping of the ejecta by interaction with the surrounding medium. In the case of genuine deviation from spherical symmetry in the explosion mechanism, an axis of symmetry that is orthogonal to that of the CSM seems unlikely. Certainly, orthogonal components were not observed in Type IIn SN 1997eg, where, likewise, Hoffman et al. (2008) found a dual axis of symmetry. The circumstellar and ejecta components in that case, however, were separated by $12^{\circ}$ not $90^{\circ}$ as is the case here. A more likely scenario for SN 2009ip is that the ejecta are forcibly elongated along the orthogonal axis due to interaction with the CSM, i.e. the circumstellar material prevents the ejecta expanding in that direction. This implies that the CSM is of a mass high enough to change the momentum of the ejecta.

The observations made in 2012 December $(\sim+60$ to $+70 \mathrm{~d})$ represent a new phase in the evolution of SN 2009ip, seen photo- metrically, spectroscopically and now observed in the polarimetric data as well. The rotation and decrease of the continuum polarization between +35 and $+68 \mathrm{~d}$ (Fig. 5) suggests that the photosphere of the ejecta changes shape from a prolate ellipsoid with long axis at $45^{\circ}$ to an oblate ellipsoid with the long axis at $\sim 120^{\circ}$. Such a change in the geometry may be the result of a jet-like explosion (Höflich, Wheeler \& Wang 1999). The change in the shape of the photosphere is coincident with a steep decline in the photospheric radius as determined by Margutti et al. (2014); indicating that in the later epochs the inner core of the explosion is revealed.

The continuum polarization at $+68 \mathrm{~d}$ of $\sim 0.5$ per cent corresponds to an axial ratio of $\sim 0.9$ or 10 per cent asymmetry, implying that the inner ejecta of the explosion are slightly more spherical than the outer envelope. The major axis of the photosphere rotates from the previous orientation at $+35 \mathrm{~d}$ to approximately align with the axis of the disc-like CSM. Note that this rotation is partly dependent upon the choice of the ISP and that no physical rotation in the ejecta is implied, instead a rotation may result from a change in the underlying energy distribution. We suggest this is the result of interaction of the ejecta with the surrounding medium leading to aspherical cooling, and thus aspherical recombination of the outer envelope in the later epochs.

The decreasing degree of polarization at low velocities, as well as the almost complete disappearance of the narrow P Cygni absorption component in the later epochs, implies that the narrow line-forming region is much smaller in relation to the size of the photosphere than it was at $+35 \mathrm{~d}$. The simulation performed in Section 5.3.2 suggests that this is the consequence of the rotation of the photosphere before $+68 \mathrm{~d}$, such that the inclined CSM blocks a much smaller region of the photosphere at this epoch.

The polarization angle for the narrow and broad absorption components of $\mathrm{H} \alpha$ remains fixed from $+35 \mathrm{~d}$. In contrast, high-velocity $\mathrm{He}_{\mathrm{I}} \lambda 5876, \mathrm{H} \beta, \mathrm{Ca}$ II and the $\mathrm{H} \alpha$ 'narrow notches' generally evolve following the continuum polarization angle. The flux spectra alone show signatures of the complex velocity structure of the ejecta observed in both the Balmer and $\mathrm{He}$ I $\lambda 5876$ lines. The polarization spectra reveal an asymmetric, shared and possibly clumpy lineforming region for these species.

\subsection{Origin of the CSM}

The observation of a disc-like structure for the CSM raises the question of its formation, whether preformed in an LBV wind, previous eruptions, through the interaction with a binary companion or, indeed, formed during the 2012 explosion itself. The formation of this disc in a cool LBV wind seems unlikely considering that the radial velocity is on the order of $\sim 1000 \mathrm{~km} \mathrm{~s}^{-1}$ which is much higher than the typical LBV terminal velocity of $\sim 200 \mathrm{~km} \mathrm{~s}^{-1}$ (Davies, Oudmaijer \& Vink 2005). Additionally, Davies et al. (2005) found evidence of clumpiness at the base of LBV winds rather than axisymmetry. It is possible, however, that the observed CSM was formed by an asymmetric explosion in one of SN 2009ip's many previous outbursts. The ejecta at $+35 \mathrm{~d}$, presuming that it was launched in 2012 July, would have caught up to material that was launched in 2011 July and August. During this period of time, the putative LBV progenitor exhibited erratic outbursts (Pastorello et al. 2013) and thus the observations here perhaps suggest a degree of asymmetry for these eruptions.

A somewhat natural explanation for asymmetry in pre-existing CSM would be interaction with a binary companion. Soker \& Kashi (2013), Kashi et al. (2013) and Tsebrenko \& Soker (2013) have 
discussed the outbursts of SN 2009ip in the context of binary interaction between an LBV and a more compact companion which is proposed to launch jets upon accretion from the more massive object. Such interactions in the pre-explosion system could lead to a bipolar arrangement for the CSM (Mcley \& Soker 2014). Smith \& Tombleson (2015) suggested a binary rejuvenation or merger scenario for the origin of massive LBV stars in environments without large star-forming regions (although Humphreys et al. 2016 have disputed this analysis). Such an environment for SN 2009ip was confirmed by Smith, Andrews \& Mauerhan (2016b), who also attribute the 2009-2011 outbursts to close binary encounters which could be the origin of the equatorial disc observed here. Binary interactions have also been suggested as the origin of the variability in $\eta$ Carinae (see for example Soker 2001 and Smith 2011).

Alternatively, the inferred geometry may not be representative of the shape of the CSM surrounding the progenitor prior to the 2012 eruptions, but formed during the outburst itself. An asymmetric distribution of energy within a spherical construct produces an oblate structure in the plane orthogonal to that with the larger energy deposition (Höflich et al. 1999). The scaleheight (or thickness) of the resulting toroidal shape is dependent upon the asymmetry of the explosion energy distribution. In this scenario, the observed orthogonality of the CSM and ejecta symmetry axes is a natural consequence of the expansion of the bipolar ejecta of the 2012a outburst into a spherical medium. The temporal evolution of the degree and angle of continuum polarization as the ejecta interact with different portions of the CSM would depend on the resulting structure; the changing geometry of the electron-scattering surface with time and the optical depth (Höflich 1995; Höflich et al. 1999).

The disc-like structure may form part of an hourglass or bipolar nebulae as observed around $\eta$ Carinae, SN 1987A and commonly seen around LBV stars (Weis 2003; Smith et al. 2016a) of which we only observe the equatorial enhancement in the narrow absorption. Chita et al. (2008) present models that describe the formation of the hourglass structures observed around blue supergiants through a fast wind-slow wind interaction. They discuss a scenario in which a star at the onset of a blue loop phase at close-to-critical rotation ejects a dense equatorial disc (Heger \& Langer 1998). The following fast wind ejected by the blue supergiant then proceeds to sweep up the slower moving material into an hourglass structure. This follows from similar models performed by Langer, García-Segura \& Mac Low (1999) which describe the formation of the Homunculus nebula in the Great Eruption of $\eta$ Car. In that scenario, the LBV wind becomes slow and very dense during outburst, while the close-tocritical rotation results in the confinement of this wind close to the equator. An ensuing fast wind sweeps up the material into two thinshelled lobes. Groh et al. (2009) suggested that the close-to-critical rotation required in these models to eject an equatorial disc is a typical attribute of strongly active LBVs.

An LBV progenitor to SN 2009ip raises the possibility of an $\eta$ Car-like CSM with bipolar lobes in addition to the equatorial enhancement observed here. In the inclined-disc scenario, it is unclear whether or not the ejecta are still interacting with the equatorial skirt. The narrow Balmer emission lines suggest interaction is ongoing. The lack of significant reprocessing of photons by electronscattering indicates, however, that strong interaction with a dense CSM is no longer dominant. As the bipolar lobes of $\eta$ Car are much larger in extent than the equatorial disc, it is possible that the ejecta of SN 2009ip continue to interact with any CSM lobes throughout these observations. The observed narrow emission lines and possibly the 'bump' in the light curve may be produced this way. It is however, unlikely that the presence of any hourglass shape would block the photosphere in a way as to have an impact on the polarization. The bipolar lobes would be much larger in extent than the photosphere and therefore would occlude it entirely, leading to the complete cancellation of the electric vectors of the light. It is possible that, similarly to $\eta$ Car, the progenitor to $\mathrm{SN}$ 2009ip was surrounded by a Homunculus-shaped nebula, however the polarimetric observations here reveal only an equatorial density enhancement.

\subsection{Physical constraints given the geometry}

SN 2009ip has been a well-documented transient, and numerous models have been proposed in an effort to understand the observations. Three categories of models have been proposed to explain the 2012 events; a terminal SN explosion; another 'impostor' outburst of an LBV star and ensuing interaction or through close encounters and a possible merger with a companion star.

Among the non-terminal eruption models is the pulsational pair instability eruption favoured by Pastorello et al. (2013) and Fraser et al. (2013), however the model offers no prediction for the geometry of the explosion or interaction. Thus, the polarimetric data cannot aid us in distinguishing it from other models of the outbursts. Margutti et al. (2014) considered the energetics of the collision of the ejecta from the $2012 \mathrm{~b}$ event with a spherical CSM. This required an explosion energy of only $\sim 10^{49} \mathrm{erg}$, considerably lower than those required for an SN explosion. The disc-like geometry of the CSM inferred from the spectropolarimetry, however, implies that the explosion energy derived by Margutti et al. is likely to be significantly underestimated. A disc-like CSM would interact with a much smaller portion of the ejecta than a spherically symmetric CSM, therefore requiring much larger kinetic energy in the explosion in order to reproduce the observed luminosity. Mauerhan et al. (2014) estimated that only 2-3 per cent of the ejecta would intercept a disc of thickness $\sim 10 \mathrm{AU}$, the extent of the CSM necessary to explain the line intensity of $\mathrm{H} \alpha$ (Levesque et al. 2014). Instead the observations may point to the 2012 events as being the result of a terminal SN explosion of energy $\gtrsim 10^{51} \mathrm{erg}$ (Smith et al. 2014; Mauerhan et al. 2014). This argument is only valid under the assumption that the geometry observed here is a structure that existed prior to the 2012a explosion and not, as the case may be, formed during the expansion of a highly aspherical ejecta into a spherical CSM. In the latter scenario, the ejecta would encounter a much larger fractional area than it would with a pre-existing disc.

The polarimetry is indicative of a bipolar explosion for the 2012 outbursts, consistent with the predictions of the non-terminal binary interaction models of Soker \& Kashi (2013), Kashi et al. (2013) and Tsebrenko \& Soker (2013). The predicted geometry of SN 2009ip also fits in quite well with the bipolar structure commonly observed in LBV nebulae (Weis 2003). The existence of a disc (torus) around $\eta$ Car (Morris et al. 1999) implies that such circumstellar media do exist around some LBV stars, Davies et al. (2005) found no evidence for this type of structure in the winds of three LBVs. Nota et al. (1995) suggested that an equatorial density enhancement is required to create the bipolar nebulae surrounding LBVs. If SN 2009ip survived the 2012 outbursts, the observations of a probable disc (or torus) here, may be evidence of the proposed method of forming the bipolar structure.

The continuum polarization angle measured at the $2012 \mathrm{~b}$ peak $\left(72^{\circ}\right)$ is significantly different from the polarization angle of the narrow $\mathrm{H} \alpha$ component $\left(\sim 41^{\circ}\right)$, from which we determine the inclineddisc or torus structure for the CSM. The difference in the inferred orientations of the disc could indicate an evolving CSM shape, such 
as that expected from an aspherical explosion interacting with a spherical CSM. It does, however, seem unlikely that the orientation of the 2012a outburst $\left(\sim 166^{\circ}\right)$ would result in the CSM residing in the plane indicated by the low-velocity line polarization observed here. Alternatively, it is also possible that the continuum polarization at the $2012 \mathrm{~b}$ peak is the result of a pseudo-photosphere generated by the interaction of the ejecta with the inner edge of the same disc that we observe at $+35 \mathrm{~d}$ (with no discrepancy). The polarization that would arise from the interaction zones of either the pre-existing disc or the spherical CSM scenario is unclear without complex 3D modelling of the interaction.

We have shown that the continuum polarization angle is subject to the choice of ISP and to temporal evolution, rotating between the inferred orientations (from line polarization) of the ejecta and the CSM over time. The continuum polarization at any one time may not be representative of the general orientation of the ejecta or of the interaction. We therefore suggest caution when interpreting the physical implications of the continuum polarization in SN 2009ip and perhaps in all Type IIn SNe, where there may be multiple sources of continuum light.

\subsection{SN 2009ip in the context of core-collapse supernovae}

Spectroscopically, SN 2009ip has been shown to be similar to Type IIP SNe (Graham et al. 2014; Margutti et al. 2014; Mauerhan et al. 2013; Smith et al. 2014) and indeed if the underlying explosion of SN 2009ip was a true core-collapse event it is expected to resemble a Type IIP SNe. Here, we compare the polarized properties of SN 2009ip to those of Type IIP, Type IIn and to the stripped CCSNe in an effort to further classify SN 2009ip within these groups.

In general, Type IIP SNe show moderate levels of continuum polarization $(\sim 0.1-0.5$ per cent $)$ while in the plateau phase, this drastically increases to, in some cases, $\gtrsim 1$ per cent when the photosphere recedes to reveal the inner asymmetry of the explosion mechanism (Leonard et al. 2006). Stripped-core SNe have been shown to display relatively low levels of continuum polarization ( $\sim 0.4$ per cent) but line polarization levels of $\sim 4$ per cent, particularly with the Ca II IR triplet (e.g. Maund et al. 2009). The maximum continuum polarization observed with SN 2009ip is $\sim 1.7$ per cent at around the peak of the 2012b light curve (Mauerhan et al. 2014). The geometry of the photosphere, however, is likely to be governed by the interaction at this stage and not purely by the ejecta. At $+35 \mathrm{~d}$, the continuum polarization, thought to arise in the fastmoving ejecta, is found to be $\sim 0.8$ per cent. This is slightly higher than typically seen for Type IIP SNe during the plateau phase and is more similar to the levels displayed by SN 2004dj and SN 2007aa around the end of the plateau phase (Leonard et al. 2006; Chornock et al. 2010). This polarization, however, is also unlikely to represent the asymmetry of the explosion, as interaction is expected to have played a part in shaping the ejecta of SN 2009ip. Mauerhan et al. (2014) compared the polarimetric properties of SN 2009ip to those of Type IIb SN 1993J, noticing that $~ 50 \mathrm{~d}$ after peak the SNe are similar in degree of polarization and polarization angle, but that around peak SN 1993J displayed more variation around the average $p$ and $\theta$ than SN 2009ip.

If there is an underlying Type IIP SN in SN 2009ip we could expect to see a similar increase in the continuum polarization at $\sim 100 \mathrm{~d}$ after explosion (Arcavi et al. 2012). This corresponds to the geometric discontinuity observed at the end of the plateau phase in other Type IIP SNe (Leonard et al. 2006; Chornock et al. 2010; Maund et al. 2007b). Assuming that the onset of the 2012a event represents the SN explosion epoch, the beginning of our observa- tions corresponds to $\sim 100 \mathrm{~d}$. There are no indications, however, of a discontinuity in the geometry at this time, as the continuum polarization appears only to decrease between the peak of $2012 \mathrm{~b}$ (Mauerhan et al. 2014) and the beginning of these observations. Should the onset of $2012 \mathrm{~b}$ be taken as the explosion epoch the plateau phase would end around 2013 January 1, after SN 2009ip had set and our observations finished.

Spectropolarimetric observations have been performed for only a few Type IIn SNe, namely SN 1997eg, SN 1998S and SN 2010j1 (Hoffman et al. 2008; Leonard et al. 2000; Wang et al. 2001; Patat et al. 2011; Bauer et al. 2012). Similarly to SN 2009ip, the other Type IIn SNe exhibited significant continuum polarization. SNe 1997eg and 2010jl were both polarized to $p \sim 2$ per cent (Hoffman et al. 2008; Patat et al. 2011; Bauer et al. 2012), for SN 1998S, the continuum polarization increased from $\sim 2$ per cent to $p \sim 3$ per cent upon removal of the ISP (Leonard et al. 2000). Under a different estimate for the ISP, Wang et al. (2001) found continuum polarization for SN 1998S in the range of 1.6-3 per cent. The maximum continuum polarization observed in the data presented here is $\sim 0.8$ per cent at $+35 \mathrm{~d}$ and $\sim 1.7$ per cent at around the $2012 \mathrm{~b}$ peak as observed by Mauerhan et al. (2014); implying a lower degree of asymmetry for SN 2009ip compared to the other Type IIn SNe. Given the uncertainty in where the continuum polarization is generated and what governs the geometry of the ejecta in Type IIn SNe, it is difficult to determine if this means a greater asymmetry in the explosion mechanism or in the CSM around the other SNe. For both SN 1998S and 1997 eg, the continuum polarization is thought to be produced in the ejecta, as is the case for SN 2009ip after $+35 \mathrm{~d}$. This could imply a greater asymmetry in the explosion mechanism for those $\mathrm{SNe}$ in comparison to SN 2009ip.

For SN 2009ip, we observe no significant increase in the degree of polarization in the broad emission wings of $\mathrm{H} \alpha$ compared to the core of the broad component. This is not the case for SN 2010j1, where the degree in polarization is enhanced in the wings and interpreted as due to upscattering of the photons in a dense fully ionized CSM (Patat et al. 2011). The lack of more highly polarized wings in SN 2009ip indicates that there is little reprocessing of the photons, at least from $+35 \mathrm{~d}$, and implies a line-forming region in the fastmoving ejecta, similarly to the broad emission line-forming regions in SNe 1997eg and 1998S (Hoffman et al. 2008; Leonard et al. 2000).

The similar behaviour of the broad $\mathrm{H}$ and $\mathrm{He}_{\mathrm{I}} \lambda 5876$ lines in SN 2009ip suggests that the ejecta are both H- and He-rich, the polarization at low velocity for both of these features indicates that the surrounding medium is also $\mathrm{H}$ - and He-rich. This also seems to be the case for SN 2010jl, where helium appears to be polarized in the same manner as the broad hydrogen features, suggesting that the CSM surrounding SN 2010j1 is also helium-rich (Patat et al. 2011; Bauer et al. 2012). Hoffman et al. found that for SN 1997eg, He I $\lambda 5876$ was polarized via electron-scattering in the ellipsoidal ejecta in which the line formed. The line also behaved differently compared to the Balmer lines on the $q-u$ plane. They concluded that the ejecta for SN 1997eg were helium-rich, while the CSM was composed of hydrogen. This could imply a difference in mass loss history between the progenitors of SN 1997eg and SNe 2010j1 and 2009ip. For SN 1998S, the mass-loss history was determined to be episodic (Leonard et al. 2000) as has been observed with SN 2009ip.

For SNe 1997eg, 1998S and 2010jl, the geometry of the CSM has been inferred to be disc-like (Hoffman et al. 2008; Leonard et al. 2000; Wang et al. 2001; Patat et al. 2011; Bauer et al. 2012; Andrews et al. 2011; Chandra et al. 2015). In addition, the spectra 
of the more recent SN 2015bh, an SN 2009ip-like event, have also displayed evidence of asymmetry in the ejecta and CSM, with a disc-like geometry proposed (Elias-Rosa et al. 2016; Thöne et al. 2016; Goranskij et al. 2016). The inferred disc or torus-like CSM surrounding SN 2009ip is consistent with the geometries suggested for other Type IIn SNe. In general, the inferred geometrical configurations for each of the Type IIn SNe are similar (aspherical ejecta surrounded by a disc-like or torus CSM) however differences remain in the composition of the CSM and how it induces a polarized signal. For SN 1997eg and SN 2010jl, electron-scattering in the CSM is responsible for the observed polarization, however for SN 2009ip the equatorial disc obscures the photosphere inducing the polarized signal. The evolution of the polarimetry corroborates what other observables such as the double-peaked light curve, the observed broad lines and the sustained eruptive mass loss demonstrated: SN 2009ip is not quite the same as a typical Type IIn SN.

\section{CONCLUSIONS}

During observations covering the period from +35 to +83 d (with respect to the UV maximum of the $2012 \mathrm{~b}$ re-brightening), SN 2009ip exhibited significant continuum and line polarization. The continuum was intrinsically polarized at the $0.3-0.8$ per cent level throughout the observations, implying asphericities of $\sim 10$ 15 per cent in the shape of the photosphere. The observed degree and angle of the continuum polarization were shown to depend on the choice of the ISP, and therefore interpretation of these results should be approached with caution.

Significant polarization was found associated with the absorption profiles $\mathrm{H} \alpha, \mathrm{H} \beta, \mathrm{He}_{\mathrm{I}} \lambda 5876$ and $\mathrm{Ca}$ II IR triplet. Mirroring the total flux profile, the polarized flux of $\mathrm{H} \alpha$ was composed of multiple components. Both broad and narrow depolarization was observed to be associated with narrow and broad P Cygni emission profiles. Narrow peaks in the polarization $(\sim 1$ per cent) were associated with absorption features at low $\left(\sim-1000 \mathrm{~km} \mathrm{~s}^{-1}\right)$ and high velocities. In contrast to the behaviour of the high-velocity material, the polarization associated with the low-velocity narrow $\mathrm{H} \alpha$ P Cygni absorption exhibited no temporal evolution. The distinct polarization angles and differing behaviour revealed the independent geometries of the fast-moving ejecta and the highly aspherical, slow-moving line-forming region generated in the CSM. The similarity of polarization peaks for $\mathrm{H} \alpha, \mathrm{H} \beta$ and $\mathrm{He}$ I $\lambda 5876$ suggested a shared structure for hydrogen and helium at both high $\left(\sim-13,800 \mathrm{~km} \mathrm{~s}^{-1}\right)$ and low velocities.

A Monte Carlo simulation of the photosphere and line-forming region of the low-velocity $\mathrm{H} \alpha$ feature was performed in an effort to reproduce the geometry of the CSM. A disc-like structure observed edge-on or inclined by $14^{\circ} \pm 2^{\circ}$ to the line of sight successfully replicated the observed Stokes parameters at +35 and $+68 \mathrm{~d}$. The inclined-disc model was expected to begin to cover the photosphere, producing an absorption feature, just prior to the $2012 \mathrm{~b}$ maximum and disappear at $\sim+70 \mathrm{~d}$. This was in agreement with the observed disappearance of the absorption line between +68 and $+83 \mathrm{~d}$. The edge-on disc model was difficult to reconcile with the absence of a low-velocity P Cygni absorption during the 2012a outburst. In addition, the observation of a thin disc-like structure edge-on would require specific alignment.

The inclined disc-like CSM was thus favoured. In this scenario, the low-velocity $\mathrm{H} \alpha$ polarization is produced by the same material that interacts with the ejecta at earlier times to power the $2012 \mathrm{~b}$ maximum. We identified two distinct phases in the evolution of SN 2009ip. In the first phase at $\sim+35 \mathrm{~d}$, the disc structure induces a strong polarization by blocking the ejecta photosphere that is elongated in the plane perpendicular to the CSM. Two scenarios naturally explain the orthogonality of the ejecta and the CSM. In the case of a pre-existing disc (prior to the 2012a outburst), the ejecta will more easily expand in the orthogonal direction. Alternatively, an aspherical explosion in the 2012a outburst expanding into a spherical CSM will result in an oblate or disc-like structure in the perpendicular direction. The observations made in 2012 December $(\sim+70 \mathrm{~d})$ represent a new phase in the polarimetric data where the inner core of the explosion is revealed upon recession of the photosphere. At this stage, the photosphere rotates to align with the CSM.

The inferred geometry of SN 2009ip fits in quite well with the observed bipolar nebulae surrounding LBVs. The possibility of pre-existing bipolar lobes similar to the Homunculus nebula of $\eta$ Car is not ruled out, however the polarimetric observations here are not suggestive of the existence of such lobes. The geometrical configuration of the CSM surrounding SN 2009ip is similar to that inferred for other Type IIn SNe, however differences remain in the composition of the previously ejected material. The CSM here is both hydrogen- and helium-rich in contrast to the hydrogen-rich CSM observed around other SNe. The evolution of the narrow and broad line profiles imply that SN 2009ip is not quite a typical Type IIn.

The observation of the disc geometry for the CSM may imply the terminal explosion of the LBV progenitor in one of the 2012 outbursts, however, this depends on the origin of this geometry. The implication of terminal SN explosion energies is only valid if the disc-like CSM pre-dates the 2012a explosion. A highly bipolar explosion colliding with a spherical circumstellar shell may result in the formation of this type of structure during the 2012b event, in which case the ejecta would interact with a large fractional area of the CSM. The 2012b light curve could then be powered through interaction with a non-terminal eruption. It is not clear that the inferred orientation of the inclined disc here is consistent with that of Mauerhan et al. (2014); simulations of the interaction of the ejecta with both the disc-like CSM and an originally spherical CSM are required in order to investigate if the continuum polarization observed at the peak of the interaction can be replicated.

\section{ACKNOWLEDGEMENTS}

We thank the ESO Director General for awarding discretionary time for the observations and the observers at Paranal for acquiring these high-quality observations. This is based on observations made with ESO Telescopes at the Paranal Observatory, under programme 290.D-5006. ER was supported by a PhD studentship awarded by the Department for Employment and Learning (DEL) of Northern Ireland. The research of JRM is supported through a Royal Society University Research Fellowship. ER thanks Morgan Fraser, Cosimo Inserra and Anders Jerkstand for useful discussions. This research has made use of the NASA/IPAC Extragalactic Database (NED), which is operated by the Jet Propulsion Laboratory, California Institute of Technology, under contract with the National Aeronautics and Space Administration. We thank the anonymous reviewer for their helpful comments.

\section{REFERENCES}

Andrews J. E. et al., 2011, AJ, 142, 45

Appenzeller I. et al., 1998, The Messenger, 94, 1

Arcavi I. et al., 2012, ApJ, 756, L30 
Bauer F. E., Zelaya P., Clocchiatti A., Maund J., 2012, in Proc. IAU Symp. 279, Death of Massive Stars: Supernovae and Gamma-Ray Bursts. Kluwer, Dordrecht, p. 325

Berger E., Foley R., Ivans I., 2009, ATel, 2184

Chandra P., Chevalier R. A., Chugai N., Fransson C., Soderberg A. M., 2015, ApJ, 810, 32

Chita S. M., Langer N., van Marle A. J., García-Segura G., Heger A., 2008, A\&A, 488, L37

Chornock R., Filippenko A. V., Li W., Silverman J. M., 2010, ApJ, 713, 1363

Chugai N. N., 2001, MNRAS, 326, 1448

Crotts A. P. S., Yourdon D., 2008, ApJ, 689, 1186

Davies B., Oudmaijer R. D., Vink J. S., 2005, A\&A, 439, 1107

Elias-Rosa N. et al., 2016, MNRAS, 463, 3894

Elmhamdi A. et al., 2003, MNRAS, 338, 939

Fesen R. A., Morse J. A., Chevalier R. A., Borkowski K. J., Gerardy C. L., Lawrence S. S., van den Bergh S., 2001, AJ, 122, 2644

Filippenko A. V., 1997, ARA\&A, 35, 309

Foley R. J., Berger E., Fox O., Levesque E. M., Challis P. J., Ivans I. I., Rhoads J. E., Soderberg A. M., 2011, ApJ, 732, 32

Fox O. D. et al., 2015, MNRAS, 447, 772

Fraser M. et al., 2013, MNRAS, 433, 1312

Fraser M. et al., 2015, MNRAS, 453, 3886

Gal-Yam A. et al., 2007, ApJ, 656, 372

Goranskij V. P., Barsukova E. A., Valeev A. F., Tsvetkov D. Y., Volkov I. M., Metlov V. G., Zharova A. V., 2016, Astrophys. Bull., 71, 422

Graham M. L. et al., 2014, ApJ, 787, 163

Groh J. H. et al., 2009, ApJ, 705, L25

Heger A., Langer N., 1998, A\&A, 334, 210

Heiles C., 2000, AJ, 119, 923

Hoffman J. L., Leonard D. C., Chornock R., Filippenko A. V., Barth A. J., Matheson T., 2008, ApJ, 688, 1186

Höflich P., 1991, A\&A, 246, 481

Höflich P., 1995, ApJ, 443, 89

Höflich P., Wheeler J. C., Wang L., 1999, ApJ, 521, 179

Humphreys R. M., Weis K., Davidson K., Gordon M. S., 2016, ApJ, 825, 64

Inserra C. et al., 2016, MNRAS, 459, 2721

Jansen R. A., Jakobsen P., 2001, A\&A, 370, 1056

Kankare E. et al., 2015, A\&A, 581, L4

Kashi A., Soker N., Moskovitz N., 2013, MNRAS, 436, 2484

Langer N., García-Segura G., Mac Low M.-M., 1999, ApJ, 520, L49

Larsson J. et al., 2013, ApJ, 768, 89

Leloudas G. et al., 2015, A\&A, 574, A61

Leonard D. C., Filippenko A. V., Barth A. J., Matheson T., 2000, ApJ, 536, 239

Leonard D. C. et al., 2006, Nature, 440, 505

Levesque E. M., Stringfellow G. S., Ginsburg A. G., Bally J., Keeney B. A., 2014, AJ, 147, 23

Mackey J., Mohamed S., Gvaramadze V. V., Kotak R., Langer N., Meyer D. M.-A., Moriya T. J., Neilson H. R., 2014, Nature, 512, 282

Maeda K. et al., 2008, Science, 319, 1220

Margutti R. et al., 2014, ApJ, 780, 21

Martin J. C., Hambsch F.-J., Margutti R., Tan T. G., Curtis I., Soderberg A., 2015, AJ, 149, 9

Mauerhan J. C. et al., 2013, MNRAS, 430, 1801

Mauerhan J. et al., 2014, MNRAS, 442, 1166

Maund J. R., Wheeler J. C., Patat F., Baade D., Wang L., Höflich P., 2007a, MNRAS, 381, 201
Maund J. R., Wheeler J. C., Patat F., Wang L., Baade D., Höflich P. A., 2007b, ApJ, 671, 1944

Maund J. R., Wheeler J. C., Baade D., Patat F., Höflich P., Wang L., Clocchiatti A., 2009, ApJ, 705, 1139

Maund J. R. et al., 2010, ApJ, 722, 1162

Maza J. et al., 2009, CBET, 1928, 1

Mcley L., Soker N., 2014, MNRAS, 445, 2492

Meyer M. J. et al., 2004, MNRAS, 350, 1195

Milisavljevic D., Fesen R. A., 2013, ApJ, 772, 134

Miller A. A., Li W., Nugent P. E., Bloom J. S., Filippenko A. V., Merritt A. T., 2009, ATel, 2183

Morris P. W. et al., 1999, Nature, 402, 502

Nota A., Livio M., Clampin M., Schulte-Ladbeck R., 1995, ApJ, 448, 788

Ofek E. O., Lin L., Kouveliotou C., Younes G., Göğüş E., Kasliwal M. M., Cao Y., 2013, ApJ, 768, 47

Ofek E. O. et al., 2016, ApJ, 824, 6

Pastorello A. et al., 2013, ApJ, 767, 1

Patat F., Romaniello M., 2006, PASP, 118, 146

Patat F., Taubenberger S., Benetti S., Pastorello A., Harutyunyan A., 2011, A\&A, 527, L6

Quinn J. L., 2012, A\&A, 538, A65

Reilly E. et al., 2016, MNRAS, 457, 288

Rest A., Sinnott B., Welch D. L., Foley R. J., Narayan G., Mandel K., Huber M. E., Blondin S., 2011a, ApJ, 732, 2

Rest A. et al., 2011b, ApJ, 732, 3

Schlegel E. M., 1990, MNRAS, 244, 269

Schlegel D. J., Finkbeiner D. P., Davis M., 1998, ApJ, 500, 525

Serkowski K., Mathewson D. S., Ford V. L., 1975, ApJ, 196, 261

Silverman J. M. et al., 2013, ApJS, 207, 3

Sinnott B., Welch D. L., Rest A., Sutherland P. G., Bergmann M., 2013, ApJ, 767, 45

Smith N., 2011, MNRAS, 415, 2020

Smith N., 2014, ARA\&A, 52, 487

Smith N., Tombleson R., 2015, MNRAS, 447, 598

Smith N. et al., 2010, AJ, 139, 1451

Smith N., Mauerhan J. C., Prieto J. L., 2014, MNRAS, 438, 1191

Smith N., Andrews J. E., Mauerhan J. C., Zheng W., Filippenko A. V., Graham M. L., Milne P., 2016a, MNRAS, 455, 3546

Smith N., Andrews J. E., Mauerhan J. C., 2016b, MNRAS, 463, 2904

Soker N., 2001, MNRAS, 325, 584

Soker N., Kashi A., 2013, ApJ, 764, L6

Soker N., Kashi A., 2016, MNRAS, 462, 217

Sprinthall R., 2011, Basic Statistical Analysis, 9th edn. Pearson

Taddia F. et al., 2012, A\&A, 545, L7

Taddia F. et al., 2013, A\&A, 555, A10

Tartaglia L. et al., 2016, MNRAS, 459, 1039

Thöne C., de Ugarte Postigo A., Leloudas G., Cano Z., Maeda K., 2015, ATel, 8417

Thöne C. C. et al., 2016, A\&A, 599, A129

Tsebrenko D., Soker N., 2013, ApJ, 777, L35

van Leeuwen F., 2007, A\&A, 474, 653

Wang L., Wheeler J. C., 2008, ARA\&A, 46, 433

Wang L., Wheeler J. C., Li Z., Clocchiatti A., 1996, ApJ, 467, 435

Wang L., Howell D. A., Höflich P., Wheeler J. C., 2001, ApJ, 550, 1030

Wang L., Baade D., Patat F., 2007, Science, 315, 212

Weis K., 2003, A\&A, 408, 205

Wheeler J. C., Maund J. R., Couch S. M., 2008, ApJ, 677, 1091 
APPENDIX A: LINE POLARIZATION OF SN 2009 IP

Table A1. SN 2009ip line polarization.

\begin{tabular}{|c|c|c|c|c|c|c|c|c|c|}
\hline Phase $(\mathrm{d})^{a}$ & Species & $\lambda_{0}(\AA)$ & $\lambda(\AA)$ & $v\left(\mathrm{~km} \mathrm{~s}^{-1}\right)$ & $p_{\text {line }}($ per cent $)$ & $\sigma p_{\text {line }}($ per cent $)$ & $\theta_{\text {line }}\left({ }^{\circ}\right)$ & $\sigma \theta_{\text {line }}\left({ }^{\circ}\right)$ & Grism \\
\hline 35 & $\mathrm{H} \alpha$ & 6563 & $6300-6500$ & -12000 to -3000 & 0.34 & 0.09 & 128 & 9 & $1200 \mathrm{R}$ \\
\hline \multirow[t]{2}{*}{68} & $\mathrm{H} \alpha$ & 6563 & $6300-6500$ & -12000 to -3000 & 0.65 & 0.19 & 85 & 42 & $1200 \mathrm{R}$ \\
\hline & & & & Low-velocity features & & & & & \\
\hline 35 & $\mathrm{H} \alpha$ & 6563 & 6538 & -1150 & 0.95 & 0.28 & 34 & 9 & $1200 \mathrm{R}$ \\
\hline 35 & $\mathrm{He}_{\mathrm{I}}$ & 5876 & 5853 & -1160 & 1.4 & 0.6 & 32 & 16 & $1200 \mathrm{R}$ \\
\hline 42 & $\mathrm{H} \alpha$ & 6563 & 6543 & -900 & 1.0 & 0.4 & 50 & 11 & $1200 \mathrm{R}$ \\
\hline 42 & $\mathrm{H} \beta$ & 4861 & 4838 & -1400 & 1.1 & 0.3 & 98 & 9 & $300 \mathrm{~V}$ \\
\hline \multirow[t]{2}{*}{68} & $\mathrm{H} \alpha$ & 6563 & 6538 & -1150 & 0.9 & 0.7 & 47 & 21 & $1200 \mathrm{R}$ \\
\hline & & & & Higher velocity features & & & & & \\
\hline 64 & $\mathrm{H} \alpha$ & 6563 & 6307 & -12150 & 0.6 & 0.4 & 109 & 18 & $300 \mathrm{~V}$ \\
\hline 64 & $\mathrm{H} \alpha$ & 6563 & 6352 & -9900 & 0.7 & 0.3 & 121 & 14 & $300 \mathrm{~V}$ \\
\hline 64 & $\mathrm{He}_{\mathrm{I}}$ & 5876 & 5692 & -9700 & 0.7 & 0.3 & 124 & 13 & $300 \mathrm{~V}$ \\
\hline 64 & $\mathrm{H} \alpha$ & 6563 & 6412 & -7000 & 0.8 & 0.3 & 139 & 12 & $300 \mathrm{~V}$ \\
\hline 64 & $\mathrm{Ca}$ II & 8542 & 8408 & -4700 & 1.1 & 0.4 & 111 & 9 & $300 \mathrm{~V}$ \\
\hline 64 & $\mathrm{He}_{\mathrm{I}}$ & 5876 & 5827 & -2500 & 1.0 & 0.3 & 127 & 10 & $300 \mathrm{~V}$ \\
\hline 64 & $\mathrm{Ca}_{\text {II }}$ & 8662 & 8573 & -3100 & 0.8 & 0.4 & 95 & 14 & $300 \mathrm{~V}$ \\
\hline 73 & $\mathrm{H} \beta$ & 4861 & 4625 & -15250 & 1.4 & 0.9 & 62 & 19 & $300 \mathrm{~V}$ \\
\hline 73 & $\mathrm{He}_{\mathrm{I}}$ & 5876 & 5705 & -8900 & 1.2 & 0.9 & 104 & 22 & $300 \mathrm{~V}$ \\
\hline 73 & $\mathrm{H} \alpha$ & 6563 & 6425 & -6400 & 1.1 & 0.9 & 128 & 23 & $300 \mathrm{~V}$ \\
\hline 73 & $\mathrm{H} \beta$ & 4861 & 4760 & -6300 & 1.5 & 0.9 & 114 & 17 & $300 \mathrm{~V}$ \\
\hline 73 & $\mathrm{Ca}$ II & 8542 & 8375 & -5900 & 3.4 & 0.9 & 139 & 9 & $300 \mathrm{~V}$ \\
\hline
\end{tabular}

Note. ${ }^{a}$ Relative to the UV maximum of the $2012 \mathrm{~b}$ re-brightening on the 2012 October 3.

\section{APPENDIX B: SIMULATED POLARIZATION OF THE LOW-VELOCITY H $\alpha$ FEATURE}




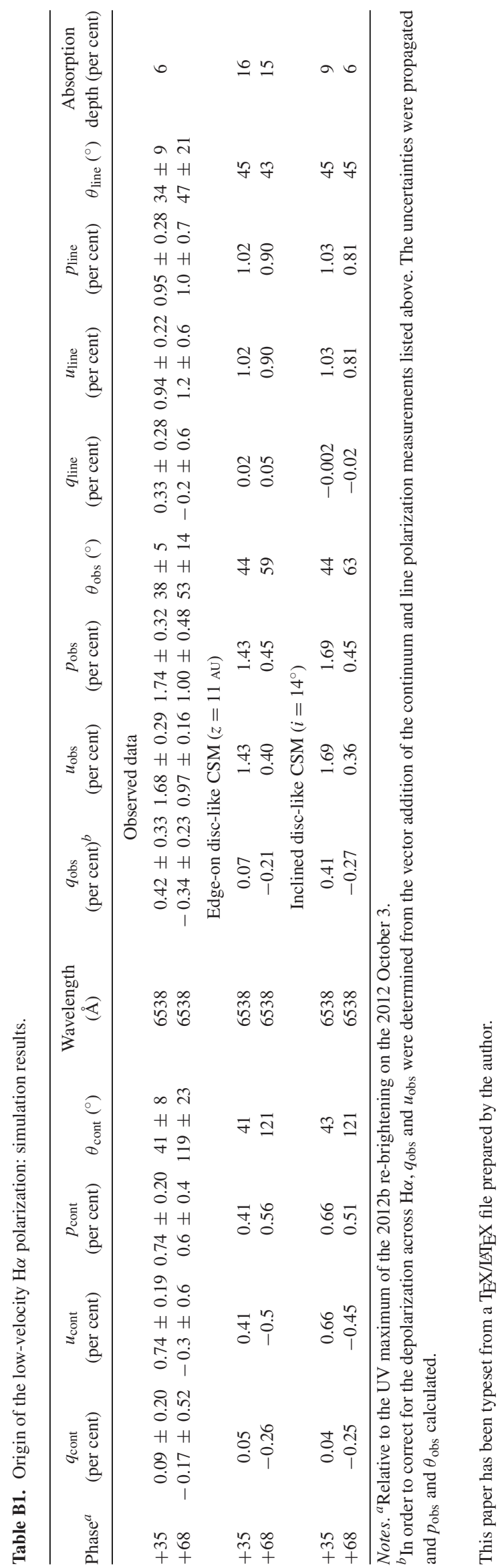

